\title{
Die Einführung der Kennzeichnungspflicht für Polizeibeamte: Ein QCA-basierter Bundesländervergleich
}

\author{
Frank Bandau $(\mathbb{D}) \cdot$ Fabio Bothner
}

Online publiziert: 2. März 2020

(C) Der/die Autor(en) 2020

Zusammenfassung Wiederholte Vorfälle von Polizeigewalt bei Demonstrationen haben in Deutschland zu Forderungen nach einer individuellen Kennzeichnung von Polizeibeamten bei derartigen Einsätzen geführt. In der Folge hat ein Teil der Bundesländer eine nummerische Kennzeichnung eingeführt, während dies in anderen Bundesländern bisher nicht geschehen ist. Der Artikel geht der Frage nach, wie diese Varianz zwischen den Ländern zu erklären ist, und ermittelt, unter welchen Bedingungen es zur Einführung der Kennzeichnungspflicht kommt. Eine crisp-set Qualitative Comparative Analysis (csQCA) dient dabei der Identifizierung von Konfigurationen von Erklärungsfaktoren, die zur Einführung der Kennzeichnung von Polizisten führen. Ergänzt wird die QCA um kurze Fallstudien (Berlin, Hessen, Mecklenburg-Vorpommern, Rheinland-Pfalz und Schleswig-Holstein), die dazu dienen, die zuvor proklamierten Kausalmechanismen zu überprüfen. Basierend auf diesem methodischen Vorgehen gelangt die Studie zu dem Ergebnis, dass die Regierungsbeteiligung von Grünen oder Linken als Agenda-Setter eine notwendige Bedingung für die Einführung der Kennzeichnung darstellt. Darüber hinaus zeigt sich, dass das Auftreten eines Agenda-Setters bei gleichzeitigem Fehlen eines Blockierers in Form von CDU/CSU eine günstige Regierungskonstellation für die Kennzeichnungspflicht darstellt. Damit es tatsächlich zur Einführung kommt, müssen aber entsprechende Rahmenbedingungen herrschen. Beim Vorreiter Berlin war dies durch das Auftreten

Zusatzmaterial online Zusätzliche Informationen sind in der Online-Version dieses Artikels (https:// doi.org/10.1007/s12286-020-00443-8) enthalten.

Dr. F. Bandau $(\bowtie) \cdot$ F. Bothner

Institut für Politikwissenschaft, Otto-Friedrich-Universität Bamberg,

Feldkirchenstraße 21, 96052 Bamberg, Deutschland

E-Mail: frank.bandau@uni-bamberg.de

F. Bothner

E-Mail: fabio.bothner@uni-bamberg.de 
eines Focusing Events der Fall, bei den Nachzüglern begünstigte der resultierende Diffusionsdruck die Durchsetzung der Kennzeichnungspflicht.

Schlüsselwörter Bundesländervergleich · Crisp-Set Qualitative Comparative Analysis (csQCA) · Kennzeichnungspflicht · Parteien · Policy-Forschung

\title{
The introduction of mandatory identification of police officers in the German Länder
}

\begin{abstract}
Recurring incidents of police violence against protesters have sparked a debate on the introduction of mandatory identification of police officers (Kennzeichnungspflicht) in Germany. In this context, some German Länder have introduced identification numbers for police officers in group operations while others have not. The article analyzes what accounts for those differences by determining the conditions under which mandatory identification is introduced. For this purpose, we combine a crisp-set Qualitative Comparative Analysis (csQCA) with short case studies. The csQCA provides configurations of conditions that lead to the introduction of mandatory identification. Case studies on five Länder (Berlin, Hesse, Mecklenburg-West Pomerania, Rhineland-Palatinate and Schleswig-Holstein) complement the QCA by testing the assumed causal mechanisms. The results are as follows: Government participation by the Greens and/or the Left Party as agenda setters is a necessary condition for the introduction of mandatory identification. In addition, government participation of an agenda setter and the simultaneous absence of an opponent, first and foremost CDU/CSU, provide favorable political conditions for introducing mandatory identification. This kind of policy change is further facilitated by favorable context factors such as focusing events, as in the case of Berlin, and rising pressure to act due to policy diffusion.
\end{abstract}

Keywords Crisp-Set Qualitative Comparative Analysis (csQCA) · German Länder · Mandatory Identification of Police Officers · Partisan Politics · Policy Analysis

\section{Einleitung}

Im Juli 2010 veröffentlichte Amnesty International den Bericht Täter unbekannt, in dem die NGO auf das Problem der Polizeigewalt in Deutschland aufmerksam machte. Als ein Kernproblem wurde in diesem Zusammenhang die Schwierigkeit der Identifizierung von Polizeibeamten im Falle von gewaltsamen Übergriffen seitens der Polizei ausgemacht (Amnesty International 2010, S. 74-78). Hauptverantwortlich für dieses Problem war laut Amnesty International die fehlende Kennzeichnungspflicht für Polizeibeamte. Als Lösung wurde das obligatorische Tragen 
von Namensschildern gefordert. Beim Einsatz geschlossener Polizeieinheiten ${ }^{1}$, also beispielsweise bei Demonstrationen, könne das Namensschild durch eine mehrstellige Nummernfolge ersetzt werden, um die Identität der Polizisten zu schützen, gleichzeitig aber die Identifizierung bei Fehlverhalten zu gewährleisten. Gegner der Kennzeichnungspflicht wie die Polizeigewerkschaften halten dem entgegen, dass eine solche Kennzeichnung überflüssig, wenn nicht gar gefährlich für die gekennzeichneten Polizeibeamten sei und außerdem ein generelles Misstrauen gegenüber der Polizei zum Ausdruck bringe (Aden 2012, S. 147-148). Seit dem Zeitpunkt der Veröffentlichung des Amnesty-Berichtes sind aus Sicht der Befürworter der Kennzeichnungspflicht deutliche Fortschritte zu verzeichnen. Gab es zu Beginn des Jahres 2010 in keinem Bundesland eine individuelle Kennzeichnungspflicht für Polizeibeamte in geschlossenen Einheiten, bestand Ende des Jahres 2018 immerhin in neun Bundesländern eine solche Kennzeichnungspflicht. ${ }^{2}$

Im Zentrum dieses Artikels steht die Frage, wie es zu erklären ist, dass einige Bundesländer in den vergangenen Jahren eine Kennzeichnungspflicht für geschlossene Polizeieinheiten eingeführt haben, während dies in anderen Bundesländern bisher nicht geschehen ist. ${ }^{3}$ Den Ausgangspunkt bei der Beantwortung dieser Frage bilden zwei Prämissen. Zum einen ist angesichts vorliegender empirischer Befunde der Policy-Forschung zur Politik der Inneren Sicherheit davon auszugehen, dass die ideologischen Differenzen zwischen Parteien einen wichtigen Erklärungsfaktor bilden (Birkel 2008; Frevel und Groß 2016; Tepe und Vanhuysse 2013; Wenzelburger 2015, S. 681-684). Zum anderen legen neuere Befunde der Policy-Forschung nahe, dass unterschiedliche Policy-Outputs zwischen den deutschen Bundesländern häufig nicht durch einzelne Faktoren wie die politische Färbung der Regierung zu erklären sind, sondern dass diese Unterschiede vielmehr aus bestimmten Kombinationen relevanter Faktoren resultieren und dass mehrere Konfigurationen zum gleichen Policy-Ergebnis führen können (Sack und Töller 2018). Die Regierungsbeteiligung bestimmter Parteien sollte demnach zwar einen Einfluss auf Policies wie die Einführung der Kennzeichnungspflicht haben - allerdings nur unter bestimmten Rahmenbedingungen.

Als methodisches Verfahren, um derartige Konfigurationen zu ermitteln, verwenden wir eine crisp-set Qualitative Comparative Analysis (csQCA), die wir um kurze Fallstudien ergänzen. Damit knüpfen wir methodisch an eine Reihe neuerer Studien an, die demonstriert haben, dass sich die QCA sehr gut für den Bundesländervergleich eignet (Beinborn et al. 2018; Günther et al. 2019; Hörisch 2018; Sack und Sarter 2018; Stoiber und Töller 2016). Auf Basis der QCA lassen sich außerdem typische sowie abweichende Fälle ermitteln (Schneider und Rohlfing 2013). Pro-

\footnotetext{
${ }^{1}$ Unter „geschlossenen Polizeieinheiten“ sind größere Verbände von Polizeibeamten zu verstehen, die besonders für das Vorgehen in derartigen Verbänden geschult sind und insbesondere bei personalintensiven Großveranstaltungen zum Einsatz kommen (Regler 2016).

2 Als zehntes Bundesland kündigte Hamburg in Folge der Vorkommnisse beim G20-Gipfel die Einführung für das Jahr 2019 an (Taz 2019).

3 Wenn im weiteren Verlauf des Artikels von der Kennzeichnungspflicht gesprochen wird, handelt es sich um die individuelle nummerische Kennzeichnung von Polizeibeamten in geschlossenen Einheiten. Davon zu unterscheiden ist die den Regelfall (z. B. im Streifendienst) darstellende Kennzeichnung durch Namensschilder.
} 
zessanalysen der typischen Fälle erlauben es, die mengentheoretischen Befunde der QCA auf ihre interne Validität zu prüfen (Blatter und Haverland 2012, S. 231). Die prozessanalytische Untersuchung abweichender Fälle ermöglicht darüber hinaus zu klären, warum diese Fälle von den zuvor ermittelten Mustern abweichen (Schneider und Rohlfing 2013, S. 565-577).

Der Artikel ist folgendermaßen aufgebaut: Der erste inhaltliche Abschnitt liefert einen Überblick zum Forschungsstand sowie eine aktuelle Übersicht zur Kennzeichnungspflicht in Deutschland. Daran schließen die theoretischen Überlegungen zu relevanten Erklärungsfaktoren für die beobachtbare Varianz zwischen den Ländern an. Nach einem kurzen Überblick über das Forschungsdesign erfolgt in Abschn. 5 die QCA, die in Kalibrierung und Präsentation der Ergebnisse unterteilt ist. Diese Ergebnisse werden in Abschn. 6 um Fallstudien zu fünf Bundesländern ergänzt. Der Artikel schließt mit einer Zusammenfassung der empirischen Befunde und einem kurzen Ausblick.

\section{Polizeipolitik und Kennzeichnungspflicht: Zum Stand der Forschung}

Nach Frevel und Groß (2016) lassen sich im Politikfeld der Inneren Sicherheit die Teilbereiche Kriminalpolitik, Justizpolitik ${ }^{4}$ und Polizeipolitik unterscheiden. Der letztgenannte Teilbereich ist für die vergleichende Policy-Forschung auf Länderebene von besonderem Interesse, da die Gesetzgebungshoheit der Länder hier mit Abstand am weitesten reicht - oder wie es Frevel und Groß auf den Punkt bringen: „Polizei ist Ländersache!“ Ein Untersuchungsgegenstand ist in diesem Zusammenhang die unterschiedliche Polizeistärke der Bundesländer, die sich neben strukturellen Faktoren wie der Bevölkerungsdichte auch auf parteipolitische Differenzen zurückführen lässt (Birkel 2008; Tepe und Vanhuysse 2013; vgl. dazu auch Frevel und Groß 2016, S. 65-66). Der parteipolitische Einfluss auf die Länderregelungen ist darüber hinaus auch in polizeirechtlichen Fragen wie finaler Rettungsschuss, Videoüberwachung und Schleierfahndung nachweisbar (Frevel und Groß 2008, S. 78). Schließlich lassen sich laut Birkel (2008) begrenzte parteipolitische Effekte auf Ebene der Policy-Outcomes nachweisen, nämlich bei der Aufklärungsquote sowie der Gefangenenrate, wobei hier die Erklärungskraft sozioökonomischer Faktoren überwiegt.

Die dem Polizeirecht zuzuordnende Kennzeichnungspflicht für Polizeibeamte wurde bisher vornehmlich aus kriminologischer und rechtswissenschaftlicher Sicht diskutiert (u.a. Aden 2010; Barczak 2011, 2014; Drescher 2014; Knaust 2017; Spitzer 2016; Thinnes 2014). Drei Studien zum Thema nehmen jedoch eine dezidiert politikwissenschaftliche Perspektive ein. Aden (2012) identifiziert in seinem Beitrag politisch relevante Befürworter und Gegner der Kennzeichnungspflicht. Unter Verwendung des ,Advocacy Coalitions'-Ansatzes arbeitet er daraufhin Akteurskoalitionen heraus, um den Erfolg oder das Scheitern von Initiativen zur Einführung der Kennzeichnungspflicht zu erklären. In einer ausführlichen Einzelfallstudie wid-

\footnotetext{
${ }^{4}$ Im Bereich der Justizpolitik beleuchten Rowe und Turner (2016) in einer aktuellen Studie die Erklärungsfaktoren für Unterschiede zwischen den Ländern in der Gefängnispolitik.
} 
Tab. 1 Kennzeichnungspflicht in Deutschland, 2010-2018

\begin{tabular}{ll}
\hline Kennzeichnungspflicht eingeführt & Kennzeichnungspflicht nicht eingeführt \\
\hline Berlin (2010) & Baden-Württemberg \\
Brandenburg (2011) & Bayern \\
Schleswig-Holstein (2012) & Hamburg \\
Rheinland-Pfalz (2013) & Niedersachsen \\
Bremen (2014) & Saarland \\
Hessen (2014) & Sachsen \\
Nordrhein-Westfalen (2016) & \\
Thüringen (2017) & \\
Sachsen-Anhalt (2017) & \\
Mecklenburg-Vorpommern (2017) & \\
\hline
\end{tabular}

Jahreszahlen in Klammern entsprechen dem Jahr des Erlasses bzw. der Verabschiedung des Gesetzes

a Abschaffung der Kennzeichnungspflicht im Jahr 2017

${ }^{\mathrm{b}}$ Einführung der Kennzeichnungspflicht im Jahr 2019

met sich Regler (2015) dem hessischen Fall, den er mithilfe des ,Multiple Streams‘-Ansatzes analysiert. In einer weiteren Studie weitet Regler (2016) den Blick, indem er nach Erklärungen für den im Bereich der Kennzeichnungspflicht zu beobachtenden länderübergreifenden Politikwandel sucht. Dabei diskutiert der Autor im Rahmen seines explorativen Vorgehens mehrere Erklärungsansätze wie Focusing Events, den Einfluss von Polizeigewerkschaften, die öffentliche Meinung und vor allem Parteiendifferenzen. Diese Studie bildet damit einen guten Ausgangspunkt für eine systematische Analyse der Konfigurationen, die zu einer Einführung der Kennzeichnungspflicht führen.

Bevor zur Betrachtung potentieller Erklärungsfaktoren übergegangen werden kann, ist eine kurze Bestandsaufnahme notwendig. Obwohl die Kennzeichnungspflicht seit den 1970er Jahren auf Landesebene immer wieder diskutiert wurde (Aden 2012, S. 143), dauerte es bis 2010, bis es erstmals zu einem entsprechenden Erlass kam. Die Vorreiterrolle nahm dabei Berlin ein, Brandenburg folgte kurze Zeit später (Regler 2016, S. 50-51). Seitdem haben insgesamt zehn Bundesländer eine Kennzeichnungspflicht für geschlossene Polizeieinheiten eingeführt, während dies in den restlichen sechs Bundesländern bisher nicht geschehen ist (siehe Tab. 1). In Hamburg ist die Einführung der Kennzeichnungspflicht für 2019 geplant, während Nordrhein-Westfalen das einzige Bundesland ist, in dem diese bereits wieder abgeschafft wurde. Die Durchsetzung der Kennzeichnungspflicht erfolgte entweder durch Dienstverordnungen der jeweiligen Innenbehörde oder per Gesetz, d.h. mit Zustimmung des jeweiligen Landtages (Aden 2010; Regler 2016, S. 50-55). Da die Einführung in jedem Fall nur mit Zustimmung aller Koalitionspartner zustande kam, können diese prozessualen Unterschiede in der empirischen Analyse jedoch vernachlässigt werden.

\section{Theoretische Überlegungen}

Anknüpfend an die genannte Literatur sowie auf der Basis eigener theoretischer Überlegungen werden in diesem Abschnitt mögliche Erklärungsfaktoren für die aufgezeigte Varianz in Bezug auf die Kennzeichnungspflicht diskutiert. Hinsichtlich 
der Erklärungsfaktoren muss zwischen Akteuren und Rahmenbedingungen unterschieden werden. Auf der Akteursebene konzentrieren wir uns auf Parteien, da die Einführung der Kennzeichnungspflicht nur mit Zustimmung der Regierungsparteien möglich ist. ${ }^{5}$ Zunächst werden daher die Positionen der deutschen Parteien zur Kennzeichnungspflicht behandelt. Daran anschließend werden mit Focusing Events und Policy-Diffusion zwei Faktoren thematisiert, die den Kontext des Regierungshandelns bilden. Dementsprechend lassen sich konfigurative Hypothesen zum Zusammenspiel von Parteien und den genannten Kontextfaktoren formulieren.

Parteien sind als Regierungsvertreter die zentralen Akteure bei der Einführung der Kennzeichnungspflicht. Grundsätzlich spielt für die Politik der Inneren Sicherheit die klassische sozioökonomische Links-Rechts-Achse eine untergeordnete Rolle. Von Relevanz ist vielmehr die Positionierung der Parteien auf der zwischen Liberalismus und Autoritarismus aufgespannten Konfliktachse (Wenzelburger 2015, S. 681-684). Auf dieser Konfliktachse bilden Grüne und Linke den liberalen Pol, während CDU/CSU (und noch mehr die AfD) auf der Gegenseite stehen. Die SPD ist weiter in der Mitte positioniert als auf der sozioökonomischen Achse, die FDP als traditionelle Bürgerrechtspartei dagegen weiter links als in Verteilungsfragen (siehe z. B. Spier 2011). Das Bild, das sich basierend auf den bisherigen Studien zur Kennzeichnungspflicht ergibt, deckt sich größtenteils mit der allgemeinen Positionierung der Parteien: Die Grünen sowie die Linke können als Kritiker von Polizeigewalt und Anhänger einer „,bürgernahen Polizei“ eindeutig dem Lager der Befürworter der Kennzeichnungspflicht zugerechnet werden, während CDU/CSU als traditionelle Verfechter von ,Law and Order' dem Lager der Gegner angehören (Aden 2012, S. 146-149). ${ }^{6}$ Für SPD und FDP ergibt sich dagegen ein differenzierteres Bild. Die SPD hat ihre, auch mit der Nähe zu den Polizeigewerkschaften begründete, vormals eher ablehnende Haltung aufgegeben. Die gegenwärtige Haltung der Landesparteien scheint stark von der Positionierung des jeweiligen Regierungspartners geprägt:

In Ländern, in denen die SPD in einer großen Koalition regiert, votiert die Partei meist klar gegen eine Kennzeichnungspflicht. [...] Ganz anders dort, wo ein SPD-Landesverband mit den Grünen oder den Linken die Regierung bildet. Hier tolerieren bzw. unterstützen die Sozialdemokraten das Vorhaben. (Regler 2016, S. 57)

Die FDP-Landesverbände sprechen sich entgegen der oben geäußerten Erwartung in Regierungsverantwortung zumeist gegen eine Kennzeichnungspflicht aus (Regler 2016), einzelne Landesverbände wie die Hamburger FDP plädieren dagegen für eine Einführung (FDP Hamburg 2018). Angesichts der insgesamt unklaren Haltung von

\footnotetext{
5 Aden (2012) thematisiert außerdem die Rolle der Polizeigewerkschaften als strikte Gegner der Kennzeichnungspflicht. Weil hinsichtlich der Stärke der Polizeigewerkschaften eine Varianz zwischen den Ländern nur schwer messbar ist und sich ihr Einfluss ohnehin fast ausschließlich über ihre Einwirkung auf potentielle parteiliche Vetospieler wie CDU/CSU und SPD bemerkbar macht (Regler 2016, S. 55-56), haben wir uns dazu entschieden, diesen Akteur in der QCA nicht zu berücksichtigen und den Fokus auf die Parteien zu legen. In den anschließenden Fallstudien wird ihrer Rolle im politischen Prozess jedoch Rechnung getragen.

${ }^{6}$ Das gilt in noch stärkerem Maße für die AfD (Regler 2016, S. 57), die aber bisher an keiner Landesregierung beteiligt war.
} 
Sozialdemokraten und Liberalen gelangen wir auf der Ebene der Parteien zu zwei Hypothesen:

H1 Die Regierungsbeteiligung der Grünen und/oder der Linken ist eine notwendige Bedingung für die Einführung der Kennzeichnungspflicht.

H2 Der Ausschluss von CDU/CSU von der Regierungsverantwortung ist eine notwendige Bedingung für die Einführung der Kennzeichnungspflicht.

Den ersten kontextuellen Erklärungsfaktor bilden sogenannte Focusing Events. Darunter sind Ereignisse zu verstehen, die ein Thema für einen gewissen Zeitraum auf die politische Agenda bringen (Kingdon 1995, S. 94-100). Im Fall der Kennzeichnungspflicht sind dies insbesondere aufsehenerregende Fälle von Polizeigewalt, beispielsweise bei Demonstrationen oder auch beim Einsatz von Spezialeinsatzkommandos (Aden 2012, S. 144-146). Jüngere Beispiele hierfür sind die Fernsehbilder von Polizeigewalt bei ,Stuttgart 21 ' und beim G20-Gipfel in Hamburg. Regler (2016, S. 55) argumentiert überzeugend, dass derartige Ereignisse als eine Art Katalysator wirken können und in einigen Bundesländern zur Einführung der Kennzeichnungspflicht beitrugen, dass sie aber - wie der Fall Baden-Württemberg zeige - alleine nicht ausreichen, um zu diesem Ergebnis zu führen. Vielmehr ist es das Zusammenspiel des Focusing Events mit den politischen Machtverhältnissen, das den PolicyOutput bestimmt:

H3 In der Folge eines Focusing Events kommt es zur Einführung der Kennzeichnungspflicht, wenn zu diesem Zeitpunkt Grüne und/oder Linke an der Regierung beteiligt sind und CDU/CSU nicht an der Regierung beteiligt sind.

Ein zweiter Prozess, der die Einführung der Kennzeichnungspflicht durch deren politische Unterstützer begünstigen sollte, ist Policy-Diffusion, also die durch die erstmalige Adaption einer Policy ausgelöste Ausbreitung dieser Policy über Ländergrenzen hinweg (Braun und Gilardi 2006; Jahn 2015). Für einen solchen Diffusionsprozess spricht, dass die Kennzeichnungspflicht sich nach der Einführung in Berlin relativ schnell in anderen Bundesländern ausbreitete. Einige Forscher sehen dementsprechend bei der Kennzeichnungspflicht ein „Schneeballprinzip“ am Werk, „,bei dem ein Land vorangeht und dann viele oder alle anderen Länder folgen“ (Frevel und Groß 2016, S. 80). Als Ursachen für Policy-Diffusion sind im vorliegenden Fall vor allem Nachahmung und Lernen denkbar, also eine eher normengeleitete Übernahme der Policy bzw. eine Übernahme der Policy aufgrund positiver Erfahrungen in anderen Bundesländern (Jahn 2015, S. 254-258). ${ }^{7}$ Beim Vorliegen von Policy-Diffusion ist davon auszugehen, dass das Auftreten eines Focusing Events nicht mehr notwendig ist.

\footnotetext{
7 Wenn immer mehr Länder die Kennzeichnungspflicht einführen, kann es außerdem zu einem steigenden Anpassungsdruck kommen, weil es gerade bei Demonstrationen und anderen Großereignissen regelmäßig zur Entsendung von Polizeieinheiten in andere Bundesländer kommt.
} 
H4 Unter Diffusionsdruck kommt es auch ohne Focusing Event zur Einführung der Kennzeichnungspflicht, wenn Grüne und/oder Linke an der Regierung beteiligt sind und CDU/CSU nicht an der Regierung beteiligt sind.

Unsere theoretischen Überlegungen lassen sich folgendermaßen zusammenfassen: Die Regierungsbeteiligung von Grünen und/oder Linken stellt ebenso eine notwendige Bedingung für die Einführung der Kennzeichnungspflicht dar wie die Abwesenheit der CDU/CSU von der Regierung. Ob es tatsächlich dazu kommt, ist allerdings von Kontextfaktoren abhängig, denen wir im Rahmen zweier konfigurativer Hypothesen Rechnung tragen.

\section{Forschungsdesign}

Die Studie analysiert die Einführung der Kennzeichnungspflicht in den deutschen Bundesländern von 2009, dem Jahr vor der erstmaligen Einführung in einem Bundesland, bis 2018. Dabei kombinieren wir eine länderübergreifende QCA mit fallspezifischen Prozessanalysen (Schneider und Rohlfing 2013). Im ersten Schritt wird mittels einer crisp-set QCA untersucht, unter welchen Bedingungen es zu einer Einführung der Kennzeichnungspflicht kommt. Die Untersuchungseinheiten bilden dabei die einzelnen Landesregierungen von 2009-2018 bzw. bis zur Einführung der Kennzeichnungspflicht (insgesamt 39 Fälle). Auf Basis der QCA werden in einem zweiten Schritt auf Ebene der Bundesländer vier typische Fälle (Berlin, SchleswigHolstein, Rheinland-Pfalz und Hessen) sowie ein abweichender Fall (MecklenburgVorpommern) identifiziert. Diese werden im Rahmen kurzer Einzelfallstudien genauer untersucht, um die fallübergreifenden Befunde der QCA mittels Prozessanalysen auf ihre interne Validität zu prüfen und das Zustandekommen des abweichenden Falles zu erklären.

\section{Crisp-set QCA: Notwendige Bedingungen und hinreichende Lösungsterme}

Die Methode der Qualitative Comparative Analysis erlaubt es, hinreichende und notwendige Bedingungen für das Auftreten eines Outcomes ${ }^{8}$ zu identifizieren. In der Forschung gibt es gegenwärtig zwei prominente Ausprägungen der QCA. Zum einen die ursprüngliche crisp-set QCA (csQCA), die mengentheoretisch binär zwischen 0 (,Nichtzugehörigkeit zu einer Teilmenge“) und 1 (,Zugehörigkeit zu einer Teilmenge") unterscheidet (Schneider und Wagemann 2012, S. 24). Zum anderen die sogenannte fuzzy-set QCA (fsQCA), welche zusätzlich graduelle Differenzierungen zwischen 0 und 1 zulässt. Da das Outcome sowie die überwiegende Mehrheit der Konditionen in binärer Form vorliegen, findet in dieser Studie die ursprüngli-

\footnotetext{
${ }^{8}$ In der Terminologie der Policy-Forschung gehören die Verordnungen bzw. Gesetze zur Einführung der Kennzeichnungspflicht zum „Policy-Output“, während diese im Rahmen der QCA das zu untersuchende „Outcome“ darstellen.
} 
che csQCA Anwendung. Im Rahmen der QCA sind die Gütemaße der Konsistenz sowie der Abdeckung von zentraler Bedeutung. Sie geben an, inwieweit eine Bedingung (auch Kondition genannt) hinreichend bzw. notwendig für das Auftreten des Outcomes ist (Schneider und Wagemann 2012, S. $123 \mathrm{ff}$.). Bei der Analyse von hinreichenden Bedingungen ist es möglich, dass eine Kombination von Konditionen als sogenannter Lösungsterm auftritt. Nicht eine Kondition für sich alleine ist dann hinreichend, sondern erst die Kombination zweier oder mehrerer Bedingungen führt zum Auftreten des Outcomes. Häufig finden sich im Rahmen der Analyse mehrere Lösungsterme, die gegebenenfalls minimiert werden können, um eine weniger komplexe Lösung zu erzielen (Ragin 2000; Schneider und Wagemann 2012, S. 104 ff.). Bei der Analyse von notwendigen Bedingungen können dagegen immer nur einzelne Konditionen als notwendig identifiziert werden (Schneider und Wagemann 2012, S. 72-73).

Um die Policy-Varianz zwischen Bundesländern zu erklären, hat die QCA in den letzten Jahren vermehrt Anwendung gefunden (Beinborn et al. 2018; Günther et al. 2019; Hörisch 2018; Sack und Sarter 2018; Stoiber und Töller 2016). Dies erscheint vor dem Hintergrund sinnvoll, dass die QCA im Gegensatz zu herkömmlichen statistischen Methoden für mittlere Fallzahlen geeignet ist. Die Stärke der QCA besteht zum einen in der Identifizierung notwendiger und hinreichender Bedingungen mithilfe der Mengentheorie. Zum anderen erweist es sich als großer Vorteil gegenüber anderen Verfahren, dass die QCA den Fokus auf Konfigurationen von Faktoren und Äquifinalität legt (Hörisch 2018; Sack und Töller 2018). Dadurch eignet sie sich ausgezeichnet, um die unterschiedlichen Kombinationen von politischen Faktoren und Kontextfaktoren zu identifizieren, die mit einer Einführung der Kennzeichnungspflicht einhergehen. Im Rahmen unserer Studie nutzen wir darüber hinaus eine weitere Stärke der QCA, nämlich dass sich auf ihrer Basis typische und abweichende Fälle identifizieren lassen, die fallspezifischen Prozessanalysen unterzogen werden können (Blatter und Haverland 2012, S. 231-235; Schneider und Rohlfing 2013).

\subsection{Kalibrierung}

Die QCA macht es notwendig, dass das Outcome sowie die einzelnen Faktoren zunächst operationalisiert und anschließend kalibriert werden. Beim Kalibrierungsprozess der QCA werden den Rohdaten Mitgliedswerte von 0 oder 1 zugewiesen. Diese Werte geben an, ob ein Fall hinsichtlich eines bestimmten Charakteristikums einer Teilmenge von Fällen angehört. Dabei bedeutet der Wert 1 jeweils eine $\mathrm{Zu}$ gehörigkeit zu einer Teilmenge, während der Wert 0 angibt, dass ein Fall einer Teilmenge nicht zugehörig ist (Schneider und Wagemann 2012). Im Folgenden stellen wir die unserer Analyse zugrunde liegende Operationalisierung und Kalibrierung der einzelnen Faktoren vor.

\subsubsection{Kennzeichnungspflicht für Polizeibeamte}

Für jede der abgedeckten 39 Legislaturperioden auf Länderebene wird erhoben, ob es in dieser Zeit zur Einführung der Kennzeichnungspflicht kam (1) oder nicht (0). 
Da das Outcome bereits in einer binären Ausprägung vorliegt, ist eine weitere Kalibrierung nicht notwendig.

\subsubsection{Parteien}

Der Einfluss von Parteien wird anhand der Regierungsbeteiligung operationalisiert. Wie bereits gezeigt, lässt sich innerhalb der Parteienlandschaft kein eindeutiges Kontinuum erkennen (vor allem SPD und FDP positionieren sich nicht eindeutig). Daher bietet es sich an, den Einfluss von Parteien anhand von zwei Konditionen abzubilden. Die erste Kondition erfasst dabei, ob eine Partei an der Landesregierung beteiligt ist, die eine Kennzeichnungspflicht entschieden ablehnt und diese damit blockieren kann. Das wäre der Fall, wenn CDU oder CSU der Regierung angehören. Die zweite Kondition gibt an, ob eine Partei in der Regierung vertreten ist, die zu den Befürwortern der Kennzeichnungspflicht gezählt werden kann, namentlich die Grünen oder die Linke. Beide Konditionen werden dichotom kalibriert und geben somit an, ob an der Regierung ein Agenda-Setter für die Kennzeichnungspflicht (Grüne, Linke) beteiligt ist und/oder ob sich in der Regierung ein Blockierer der Kennzeichnungspflicht (CDU, CSU) befindet.

\subsubsection{Focusing Events}

Die Bestimmung von Focusing Events gestaltet sich angesichts der Vielzahl möglicherweise relevanter Vorfälle als schwierig. Es stellt sich somit die Frage nach einer geeigneten Methode, um Focusing Events zu identifizieren. Unser Ansatz besteht darin, ein Focusing Event über dessen mediale und öffentliche Präsenz zu erfassen. Das Web-Tool ,Google Trends ‘ ermöglicht es, die Anfragehäufigkeit eines Suchbegriffes im Zeitverlauf abzubilden. Das Tool liefert dabei keine absoluten Zahlen, sondern normiert die Werte relativ zum höchsten absoluten Wert, die Skala reicht folglich von 0-100. Da die Kennzeichnungspflicht häufig im Zuge von ausschweifender Polizeigewalt gegen Demonstranten gefordert wird, entscheiden wir uns für den Suchbegriff „Polizeigewalt“. ${ }^{9}$ Die Suche wird auf den Zeitraum von Januar 2009 bis November 2018 eingegrenzt.

Wie in Abb. $1 \mathrm{zu}$ sehen ist, ergeben sich mehrere Zeitpunkte mit überdurchschnittlich hohen Werten. Diese Anfragespitzen zeigen an, wann die Salienz des Themas besonders hoch war, und liefern damit ein gutes Indiz für das Vorliegen eines Focusing Events. ${ }^{10}$ Um tatsächliche Focusing Events zu identifizieren, wird eine Webrecherche für die einzelnen Zeitpunkte durchgeführt. Ein Focusing Event liegt dann vor, wenn es im Zuge von Demonstrationen und vergleichbaren Veranstaltungen, bei denen Polizeibeamte ohne Kennzeichnung eingesetzt wurden, zu

\footnotetext{
9 ,Google-Trends' erlaubt neben der Suche nach einzelnen Begriffen auch eine Themensuche, welche verschiedene vordefinierte Begriffe zu einem Themenkomplex umfasst. Die Ergebnisse beider Suchen sind beim Begriff „Polizeigewalt“ nahezu identisch.

10 Mithilfe von ,Google Trends‘ kann lediglich die digitale Präsenz eines Themas erfasst werden. Da sich die Informationsgewinnung sowie -vermittlung zunehmend digital gestaltet und ,Google ‘ die größte Online-Suchmaschine darstellt, halten wir dieses Vorgehen für geeignet, um die Salienz eines Themas approximativ zu bestimmen.
} 


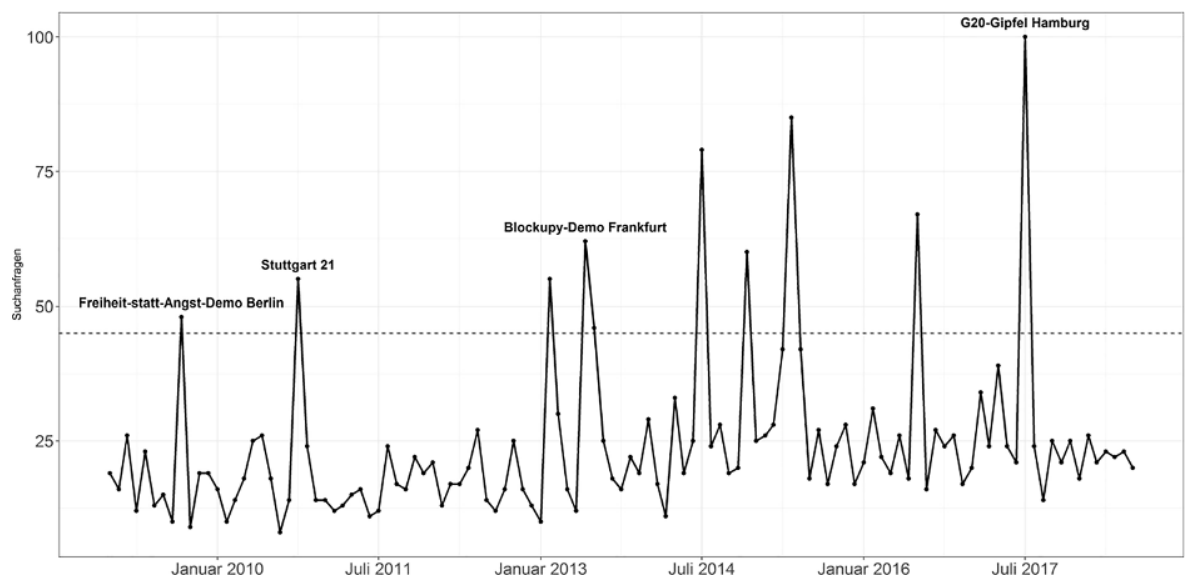

Abb. 1 ,Google Trends'-Suche nach dem Begriff „Polizeigewalt“, 2009-2018 (,Google Trends“-Suche für den Suchbegriff „Polizeigewalt“. Region: Deutschland, Zeitraum: 1. Januar 2009-30. November 2018. Die unbeschrifteten Spitzen können nicht in Zusammenhang mit Polizeigewalt bei Großereignissen in Deutschland gebracht werden [für mehr Informationen zu diesen Spitzen siehe Online-Anhang, Tab. 6])

einem gewaltsamen Vorgehen der Polizei gekommen ist. Insgesamt können auf diese Weise vier Focusing Events identifiziert werden: die Demonstration „Freiheit statt Angst“ in Berlin (2009), die Proteste gegen ,Stuttgart 21 '(2010), die ,Blockupy“Proteste in Frankfurt (2013) und der G20-Gipfel in Hamburg (2017). Die anderen Anfragespitzen konnten entweder nicht in Zusammenhang mit Polizeigewalt bei Großveranstaltungen gebracht werden oder hingen mit Vorfällen im Ausland zusammen (z.B. Proteste in den USA). Ein Effekt wird jeweils für das Bundesland angenommen, in dem das Focusing Event auftritt. ${ }^{11}$ Weil der Einfluss eines Focusing Events zeitlich begrenzt ist, beschränken wir den Effekt auf die Legislaturperiode, in der das Focusing Event auftritt (sofern der Zeitpunkt nicht nach der Einführung der Kennzeichnungspflicht liegt) sowie auf die folgende Legislaturperiode, falls diese nicht später als ein Jahr nach dem Focusing Event beginnt. Wie bei den Konditionen zuvor handelt es sich bei Focusing Events um eine dichotome Kondition, die die Ausprägung 1 für das Auftreten und die Ausprägung 0 für das Ausbleiben eines Events annimmt.

\subsubsection{Policy-Diffusion}

Bei der Bedingung der Policy-Diffusion gilt es, einen Zeitpunkt festzulegen, ab dem Landesregierungen in ihren Reformbemühungen auf die Erfahrungen mit der Kennzeichnungspflicht in anderen Bundesländern verweisen können. Wir gehen davon

\footnotetext{
11 Diese Beschränkung auf das betroffene Bundesland hat zwei Gründe: Erstens gehen wir davon aus, dass die Vorfälle trotz der bundesweiten Aufmerksamkeit vor allem im betroffenen Bundesland einen starken politischen Reformdruck erzeugen. Zweitens erwies sich eine zusätzliche ,Google Trends'-Suche auf Länderebene aufgrund der zeitlichen Instabilität der Ergebnisse als ungeeignet, um die unterschiedliche Stärke der Effekte der Focusing Events in den einzelnen Bundesländern zu identifizieren.
} 
Tab. 2 Kalibrierung der Konditionen

\begin{tabular}{|c|c|}
\hline Outcome \& Konditionen & Crisp-set-Werte \\
\hline $\begin{array}{l}\text { Outcome: } \\
\text { Kennzeichnungspflicht } \\
\text { für Polizeibeamte }\end{array}$ & $\begin{array}{l}0=\text { „keine Verabschiedung der Kennzeichnungspflicht““ } \\
1=, \text {,Verabschiedung der Kennzeichnungspflicht““ }\end{array}$ \\
\hline $\begin{array}{l}\text { 1. Kondition: } \\
\text { Blockierer }\end{array}$ & $\begin{array}{l}0=\text {,keine Regierungspartei tritt als Blockierer auf“" } \\
1=\text {,eine oder mehrere Regierungsparteien treten als Blockierer auf““ }\end{array}$ \\
\hline $\begin{array}{l}\text { 2. Kondition: } \\
\text { Agenda-Setter }\end{array}$ & $\begin{array}{l}0=\text {,keine Regierungspartei tritt als Agenda-Setter auf“" } \\
1=\text {,eine oder mehrere Regierungsparteien treten als Agenda-Setter auf““ }\end{array}$ \\
\hline $\begin{array}{l}\text { 3. Kondition: } \\
\text { Focusing Event }\end{array}$ & $\begin{array}{l}0=, \text {,kein Focusing Event“" } \\
1=, \text { Focusing Event“ }\end{array}$ \\
\hline $\begin{array}{l}\text { 4. Kondition: } \\
\text { Diffusion }\end{array}$ & $\begin{array}{l}\leq 2010=0, \text {,kein Diffusionsdruck“" } \\
>2010=1 \text {,Diffusionsdruck“" }\end{array}$ \\
\hline
\end{tabular}

aus, dass die erstmalige Verabschiedung in einem Bundesland von zentraler Bedeutung ist, da dies eine Signalwirkung entfaltet, die die Reformbefürworter in anderen Ländern dazu ermutigt nachzuziehen. Im Fall der Kennzeichnungspflicht stellt Berlin mit der Verabschiedung einer entsprechenden Dienstverordnung im Jahre 2010 den Vorreiter dar. Reformbereite Landesregierungen können somit spätestens ab 2011 auf ein Vorbild verweisen. Der Beschluss zur Einführung der Kennzeichnungspflicht wird in der Regel im Koalitionsvertrag vereinbart, also am Anfang der Legislaturperiode. Dementsprechend gehen wir für alle Legislaturperioden, die 2011 oder später beginnen, von Diffusionsdruck aus, weshalb sie den Wert 1 erhalten. Alle Legislaturperioden, die 2010 oder früher beginnen, werden dagegen mit dem Wert 0 codiert (siehe Tab. 2). Allerdings ist auch die Annahme, dass sich Diffusionsdruck erst später bemerkbar macht, nicht unplausibel, sei es weil mehr Länder die Policy verabschiedet haben oder weil es erste positive Erfahrungen mit der Umsetzung gibt. Als alternative Zeitpunkte für das Auftreten von Diffusionsdruck wählen wir daher die Jahre 2012 und 2014, um die Robustheit unserer Ergebnisse zu prüfen.

\subsection{Ergebnisse und Diskussion}

Wie weiter oben beschrieben, ermöglicht die QCA die Identifizierung von notwendigen und hinreichenden Bedingungen. Hierzu werden Schwellenwerte für die Konsistenz festgelegt. Der übliche Wert für notwendige Bedingungen liegt bei 0,9; während für hinreichende Bedingungen ein Wert von mindestens 0,75 empfohlen wird (Schneider und Wagemann 2012). Für das weitere Vorgehen wird das R-Package ,QCA“ von Dusa (2017) verwendet.

Die Analyse der notwendigen Bedingungen ergibt, dass die Kondition ,AgendaSetter" einen Konsistenzwert von 0,9 erreicht (siehe Anhang, Tab. 3). Ein Blick in die ,Truth Table‘ zeigt, dass lediglich in einem Bundesland (Mecklenburg-Vorpommern) eine Kennzeichnungspflicht für Polizeibeamte eingeführt wurde, ohne dass ein als Agenda-Setter geltender Akteur an der Regierung beteiligt war (siehe Anhang, Tab. 4). In allen anderen Fällen war ein solcher Agenda-Setter vorhanden. Neben dem Konsistenzwert sollten bei der Analyse von notwendigen Bedingungen auch der Abdeckungs- sowie der RoN-Wert (Relevance of Necessity) beachtet werden. Bei- 
de Maße liefern Anhaltspunkte, inwieweit eine Kondition als tatsächlich notwendig angesehen werden kann oder als triviale notwendige Bedingung gelten muss (vgl. Schneider und Wagemann 2012). ${ }^{12}$ Unsere Ergebnisse zeigen einen mittleren Wert für die Abdeckung $(0,529)$ und einen relativ hohen RoN-Wert $(0,733) .{ }^{13}$ Die QCA liefert damit eine Bestätigung von $\mathrm{H} 1$, wonach die Regierungsbeteiligung mindestens einer Agenda-Setter-Partei, also der Grünen oder der Linken, eine nicht triviale notwendige Bedingung für die Einführung einer Kennzeichnungspflicht darstellt. Die Analyse zeigt außerdem, dass das Nicht-Auftreten eines Blockierers lediglich einen Konsistenzwert von 0,7 für eine notwendige Bedingung erreicht. Insgesamt war die CDU in drei Ländern (Hessen, Mecklenburg-Vorpommern und Sachsen-Anhalt) an der Regierung beteiligt, während es zur Verabschiedung der Kennzeichnungspflicht kam. Somit wird H2 zum Einfluss der politischen Gegner der Kennzeichnungspflicht nicht bestätigt. Die Verbannung von CDU/CSU in die Opposition stellt demnach keine notwendige Bedingung für die Einführung der Kennzeichnungspflicht dar.

Während die Regierungsbeteiligung eines Agenda-Setters notwendig ist, verdeutlichen die Ergebnisse auch, dass dies nicht hinreichend für die Einführung der Kennzeichnungspflicht ist. Die Einführung ist vielmehr vom gemeinsamen Auftreten des Agenda-Setters mit bestimmten Kontextfaktoren abhängig. So ergibt die Analyse von hinreichenden Bedingungen drei Lösungsterme, welche verschiedene Kombinationen von Konditionen aufweisen (siehe Abb. 2). ${ }^{14}$ Der erste Lösungsterm (P1) beschreibt einen Fall (Berlin 2006-11) und zeigt, dass es zu einer Einführung kommt, wenn kein Gegner der Kennzeichnungspflicht, also weder CDU noch CSU, über Vetomacht in der Regierung verfügen, aber ein Agenda-Setter an der Regierung beteiligt ist und gleichzeitig ein Focusing Event (und keine Diffusion) vorliegt. Im zweiten Lösungsterm (P2), der wie P1 eine Konsistenz von 1,0 aufweist und ebenfalls einen Fall (Hessen 2013-18) beschreibt, liegen alle Konditionen vor. In der Regierung sind also ein Agenda-Setter und ein Blockierer vertreten, zudem liegen ein Focusing Event und Diffusion vor. Der Lösungsterm P3 deckt fünf Fälle ab, in denen es zur Einführung der Kennzeichnungspflicht kam (Bremen 2011-15, Nordrhein-Westfalen 2012-17, Rheinland-Pfalz 2011-16, Schleswig-Holstein 2012-17 und Thüringen 2014-18). P3 beschreibt aber auch einen Fall (Niedersachsen 2013-17), in dem es nicht zu diesem Outcome kam, weshalb der dritte Lösungsterm bei einer hohen Abdeckung von 0,5 eine Konsistenz von 0,83 aufweist. Um die Robustheit der Ergebnisse zu testen, wurde die Analyse zusätzlich auf Grundlage der alternativen Kalibrierungen für „Diffusion“ durchgeführt (Skaaning 2011) (siehe Online-Anhang, Abb. 5). Es zeigt sich, dass die Lösungsterme P2

\footnotetext{
12 Triviale notwendige Bedingungen treten vor allem auf, wenn die Konditionen ,skewed', also ungleich verteilt sind (vgl. Schneider und Wagemann 2012, S. 236 ff.).

13 Eine notwendige Bedingung kann als nicht trivial angesehen werden, wenn der RoN-Wert größer als 0,5 ist (vgl. Schneider 2018, S. 250-251).

14 Die vorgestellten Lösungsterme stellen die ,most complex solution “ dar. Eine weitere Reduzierung der Komplexität kann prinzipiell erreicht werden, indem Zusatzannahmen bezüglich der logischen Rudimente getroffen werden (siehe Ragin 2008, S. 167f.; Wagemann und Schneider 2015, S. 40; Thiem 2019). Dies ermöglicht die Berechnung einer ,intermediate ' oder ,parsimonious solution“. Die Ergebnisse sowie eine ausführliche Diskussion der intermediate sowie parsimonious solution finden sich im Online-Anhang (Abb. 3 und 4).
} 


\begin{tabular}{|c|c|c|c|}
\hline & \multicolumn{3}{|c|}{ Lösungsterme } \\
\hline & $\mathrm{P} 1$ & P2 & P3 \\
\hline \multirow{4}{*}{\multicolumn{4}{|c|}{$\begin{array}{l}\text { Blockierer } \\
\text { Agenda-Setter } \\
\text { Focusing Event } \\
\text { Diffusion }\end{array}$}} \\
\hline & & & \\
\hline & & & \\
\hline & & & \\
\hline Konsistenz & 1.00 & 1.00 & 0.83 \\
\hline PRI-Value & 1.00 & 1.00 & 0.83 \\
\hline Abdeckung & 0.10 & 0.10 & 0.50 \\
\hline Fälle & Berlin & Hessen & $\begin{array}{c}\text { Bremen; Niedersachsen; } \\
\text { Nordrhein-Westfalen; Rheinland- } \\
\text { Pfalz; Schleswig-Holstein; } \\
\text { Thüringen }\end{array}$ \\
\hline Konsistenz & \multicolumn{3}{|c|}{0.88} \\
\hline PRI-Value & \multicolumn{3}{|c|}{0.88} \\
\hline Coverage & \multicolumn{3}{|c|}{0.70} \\
\hline
\end{tabular}

Anmerkung: Nicht ausgefüllte Kreise zeigen an, dass eine Kondition auftritt, während mit “十” versehene Kreise angeben, dass eine Kondition nicht auftritt (Fiss 2011). Länder, in denen das untersuchte Outcome nicht auftritt, sind fettgedruckt.

Abb. 2 Darstellung der Lösungsterme für die Einführung der Kennzeichnungspflicht (Nicht ausgefüllte Kreise zeigen an, dass eine Kondition auftritt, während mit dem Kreuz versehene Kreise angeben, dass eine Kondition nicht auftritt (Fiss 2011). Länder, in denen das untersuchte Outcome nicht auftritt, sind fettgedruckt)

und P3 über verschiedene Kalibrierungen hinweg stabil sind, was unterstreicht, dass es sich bei diesen Termen um hinreichende Bedingungen handelt.

Mit Hilfe der drei Lösungsterme können insgesamt sieben der zehn positiven Outcomes erklärt werden. Eine genauere Betrachtung der Lösungsterme erlaubt eine Prüfung der zu Beginn aufgestellten konfigurativen Hypothesen. H3 zum Zusammenspiel von Focusing Events und der parteipolitischen Zusammensetzung der Regierung wird nur mit erheblichen Einschränkungen bestätigt. So geht die Verbindung aus Agenda-Setter, fehlendem Blockierer und Focusing Event wie P1 veranschaulicht in Berlin (und derzeit in Hamburg) tatsächlich mit der Einführung der Kennzeichnungspflicht einher. Der zweite Lösungsterm zeigt jedoch, dass die Kombination von Agenda-Setter und Focusing Event sogar trotz des Vorhandenseins eines Blockierers in der Regierung zur Einführung der Kennzeichnungspflicht führen kann. In Baden-Württemberg kam es dagegen nach 2011 trotz der vermeintlich günstigeren Konstellation (Agenda-Setter, kein Blockierer und Focusing Event) nicht zur Einführung der Kennzeichnungspflicht, die in H3 präsentierte Kombination führt also nicht zwangsläufig zur Kennzeichnung von Polizeibeamten. Laut H4 kommt es unter Diffusionsdruck auch ohne Focusing Event zur Einführung der Kennzeichnungspflicht, wenn eine günstige Regierungskonstellation vorliegt. Dies entspricht exakt dem dritten Lösungsterm, der damit eine Bestätigung für H4 liefert. Nachdem Berlin im Anschluss an ein Focusing Event mit der Kennzeichnung von Polizeibeamten vorangeschritten war (P1), waren an der Regierung beteiligte Befürworter der Kennzeichnung in zahlreichen Bundesländern in der Lage, die 
Kennzeichnungspflicht auch ohne Focusing Event durchzusetzen. Sie mussten dabei nicht den Widerstand eines Blockierers brechen, weil die CDU jeweils in die Opposition verbannt war. Die Ausnahme von der Regel stellt Niedersachsen dar, wo es unter einer rot-grünen Regierung (2013-17) nicht zur Einführung der Kennzeichnungspflicht kam.

Der Lösungsterm P2 stellt in gewisser Hinsicht einen Sonderfall dar, der sich dadurch auszeichnet, dass ein Agenda-Setter unter äußerst günstigen Kontextbedingungen operiert (Focusing Event + Policy-Diffusion), sich aber mit der Existenz eines Blockierers in der Regierung konfrontiert sieht. Konkret handelt es sich bei dem Fall, der diesem Lösungsterm angehört, um die schwarz-grüne Koalition in Hessen, unter der es 2015 zur Einführung der Kennzeichnungspflicht kam. Da es sich neben der schwarz-rot-grünen Regierung Sachsen-Anhalts (2016-18) um den einzigen Fall handelt, in dem die gleichzeitige Regierungsbeteiligung von AgendaSetter und Blockierer zur Einführung führte, lohnt es sich diesen Fall im Rahmen der Fallstudien genauer in den Blick zu nehmen.

Insgesamt lassen sich die zentralen Befunde der QCA folgendermaßen zusammenfassen: Erstens stellt das Vorhandensein eines Agenda-Setters in der Regierung eine notwendige Bedingung für die Einführung der Kennzeichnungspflicht dar, während das Fehlen eines Blockierers in der Regierung keine notwendige Bedingung darstellt. Zweitens bildet das Auftreten eines Agenda-Setters bei gleichzeitigem Fehlen eines Blockierers in der Regierung eine günstige politische Konfiguration für die Einführung der Kennzeichnungspflicht. Diese Befunde bestätigen, dass die Parteiendifferenztheorie im Falle der Kennzeichnungspflicht durchaus Erklärungskraft besitzt, allerdings nur unter Berücksichtigung des Kontextes. Drittens müssen zur Verabschiedung der Kennzeichnungspflicht nämlich zusätzlich zur genannten politischen Konstellation günstige Rahmenbedingungen in Form eines Focusing Events und/oder von Policy-Diffusion vorliegen.

\section{Bundesländer im Fokus}

Nachdem die QCA fallübergreifend mehrere Konfigurationen der Erklärungsfaktoren aufgezeigt hat, die zum Outcome führen, zielen die anschließenden Fallstudien darauf ab, das erwartete Zusammenspiel der Erklärungsfaktoren innerhalb einzelner Fälle zu untersuchen. Die Fallstudien dienen dabei der Prüfung der internen Validität der Ergebnisse der QCA mittels einer Aufdeckung der zugrunde liegenden Kausalmechanismen (Blatter und Haverland 2012, S. 208-211, 231). ${ }^{15}$ Den Empfehlungen von Schneider und Rohlfing (2013) zur Verbindung von QCA und Prozessanalyse folgend wird dementsprechend für jeden der drei Lösungsterme mindestens ein typischer Fall ausgewählt. Als typische Fälle fungieren Berlin (P1), Hessen (P2) sowie mit Schleswig-Holstein und Rheinland-Pfalz zwei Länder für P3, da dieser Lösungsterm das Gros der Fälle mit positivem Outcome beschreibt. Zusätzlich wird

\footnotetext{
${ }^{15}$ Mit den Polizeigewerkschaften wird auch eine in der QCA vernachlässigte Variable in die Betrachtung einbezogen, der Fokus liegt jedoch nicht auf der Identifizierung zusätzlicher, in den Lösungstermen nicht enthaltener Erklärungsfaktoren.
} 
Mecklenburg-Vorpommern als abweichender Fall untersucht, da es nur in diesem Bundesland zur Einführung der Kennzeichnungspflicht kam, ohne dass die notwendige Bedingung (Agenda-Setter in der Regierung) vorlag. ${ }^{16}$ Aus Sicht der QCA stellt dieser Fall ein Puzzle dar, zu dessen Lösung die Prozessanalyse beitragen kann (vgl. Schneider und Rohlfing 2013, S. 567-569).

\subsection{Berlin: Ein Focusing Event als Auslöser}

Berlin kann als Pionier im Bereich der Kennzeichnungspflicht betrachtet werden, weil es das erste Bundesland war, das die Kennzeichnung für Polizeibeamte in geschlossenen Einheiten einführte. Der zugehörige Lösungsterm P1 zeichnet sich neben der günstigen Regierungskonstellation durch die fehlende Diffusion bei gleichzeitigem Auftreten eines Focusing Events aus. Im Berliner Fall ist daher vor allem zu prüfen, ob und wie das vorliegende Focusing Event im Zusammenspiel mit politischen Faktoren zur Einführung der Kennzeichnungspflicht führte.

Die Absicht der Einführung der Kennzeichnungspflicht findet sich bereits im Koalitionsvertrag der Regierung aus SPD und Linken (damals noch PDS) für die Jahre 2001 bis 2006 wieder (SPD und PDS 2002, S. 13). Die Regierung enthielt also mit den Linken einen Agenda-Setter und keinen Blockierer. Interessanterweise kam es allerdings erst gegen Ende der darauffolgenden Legislaturperiode unter der gleichen Regierungskonstellation tatsächlich zur Umsetzung dieser Pläne. Eine befriedigende Erklärung dieser Verzögerung ist nur unter Einbeziehung zusätzlicher Erklärungsfaktoren möglich. So kam, wie zu erwarten, aus der Polizei massiver Widerstand gegen die Einführung einer individuellen Kennzeichnungspflicht (Diederichs 2009). Ein zentrales Argument war in diesem Zusammenhang das Fehlen einer solchen Regelung in anderen Bundesländern: „Dagegen spricht [...] dass es bundesweit eine individuelle Kennzeichnung von geschlossenen Einheiten nicht gibt. Die Mitarbeiter fragen sich, warum die Berliner Beamten bundesweit die einzigen sein sollen“ (Polizeipräsident D. Glietsch zit. in Tagesspiegel 2007). Der fehlende Diffusionsdruck lieferte den Gegnern also gewichtige Argumente, weshalb es zunächst nur zur Einführung einer taktischen Gruppenkennzeichnung kam, die jedoch keine individuelle Identifizierung erlaubte (Diederichs 2009). Eine Gesetzesinitiative der Grünen scheiterte Anfang 2007 am Widerstand der SPD. Die Linken äußerten ihre Zustimmung zum Vorstoß der Grünen, stellten sich aber aus Koalitionsdisziplin letztlich an die Seite der SPD (Abgeordnetenhaus Berlin 2007, S. 490-494).

Als Wendepunkt erwies sich die „Freiheit statt Angst“-Demonstration gegen staatliche Datenspeicherung im September 2009, an deren Rande es zu Polizeigewalt gegen einen Demonstranten kam. Die verantwortlichen Polizisten konnten erst im Nachhinein durch Videoaufnahmen anderer Demonstranten identifiziert werden. Das Versagen der bis dahin geltenden Legitimationspflicht zeigte sich in besonders eklatantem Maße, weil die Aufforderung zur Nennung der Dienstnummer seitens des Demonstranten den Auslöser der Polizeigewalt bildete (Heinrich 2011; Thinnes 2014, S. 19). Die politische Reaktion erfolgte nur wenige Wochen später in Form der

16 Damit handelt es sich bei Mecklenburg-Vorpommern um einen abweichenden Fall bezüglich der Konsistenz und nicht bezüglich der Abdeckung (vgl. Schneider und Rohlfing 2013, S. 567-575). 
Geschäftsanweisung ZSE Nr. 2/2009, die die Kennzeichnung zum 1. Januar 2011 verpflichtend machte (Polizeipräsident Berlin 2010). Der anhaltende Widerstand des Personalrats der Polizei sowie der Polizeigewerkschaften sorgte für eine weitere Verzögerung. Die Einführung zu Beginn des Jahres 2012 konnten die Vertreter der Polizei jedoch ebenso wenig verhindern wie die CDU als neuer Koalitionspartner der SPD (Peter 2011; Thinnes 2014, S. 19-21). ${ }^{17}$

Insgesamt bestätigt der Berliner Fall die Bedeutung eines Focusing Events in Fällen, in denen von den Befürwortern nicht auf Erfahrungen aus anderen Bundesländern zurückgegriffen werden kann: Die günstige Regierungskonstellation mit den Linken als Agenda-Setter und dem Fehlen eines Blockierers brachte das Thema auf die politische Agenda, fehlender Diffusionsdruck erschwerte jedoch lange Zeit die tatsächliche Umsetzung. Zur Einführung kam es erst nach einem Focusing Event, das die Mängel der geltenden Regelungen bei der Identifizierung gewalttätiger Polizeibeamter offenbarte. Die Polizeigewerkschaften leisteten massiven Widerstand, waren nach dem Focusing Event aber letztlich machtlos, da sie nicht über Vetomacht verfügten.

\subsection{Schleswig-Holstein und Rheinland-Pfalz: Ausbreitung der Kennzeichnungspflicht}

Der dritte Lösungsterm umfasst mit Bremen, Nordrhein-Westfalen, Rheinland-Pfalz, Schleswig-Holstein und Thüringen fünf Länder, die sich vom Berliner Fall dadurch unterscheiden, dass kein Focusing Event vorliegt, Befürworter aber auf die Erfahrungen aus Berlin (und später auch aus anderen Bundesländern) verweisen können. Ein Blick nach Schleswig-Holstein und Rheinland-Pfalz, wo es 2012 bzw. 2013 zur Verabschiedung der Kennzeichnungspflicht kam, soll daher vor allem prüfen, ob - wie von uns in der QCA unterstellt - bereits zu diesem frühen Zeitpunkt Diffusionsdruck feststellbar ist, also ob sich Agenda-Setter in den jeweiligen Landesregierungen bei ihren Initiativen auf die Erfahrungen in den Pionierländern beriefen.

In Schleswig-Holstein erfolgte ein erster Vorstoß bereits im Februar 2010, als Grüne und Linke gemeinsam einen Gesetzentwurf einbrachten (Landtag Schleswig-Holstein 2010a). Dieser Gesetzentwurf wurde nicht nur von der schwarz-gelben Regierung abgelehnt, sondern stieß auch bei der SPD und dem Südschleswigschen Wählerverband (SSW) auf Ablehnung (Landtag Schleswig-Holstein 2010b, S. 780-789). Die Landtagswahlen im Mai 2012 führten zum Regierungswechsel von Schwarz-Gelb zu einer Dreierkoalition aus SPD, Grünen und SSW, womit sich die politische Ausgangslage maßgeblich veränderte. Statt des strikten Kennzeichnungspflicht-Gegners CDU fand sich nun mit den Grünen ein Agenda-Setter in der Regierungsverantwortung. Gegen die ursprünglichen Skeptiker von SPD und SSW gelang es den Grünen, die Kennzeichnungspflicht im gemeinsamen Koalitionsvertrag zu verankern (SPD, Grüne und SSW 2012, S. 52). Neben den positiven Erfahrungen in anderen westlichen Demokratien verwiesen die Grünen dabei auf

${ }_{17}$ Bei den Koalitionsverhandlungen im Herbst 2011 erreichte die CDU lediglich einen regelmäßigen Austausch der Kennziffern (SPD und CDU 2011, S. 67). 
die erfolgreiche Einführung der Kennzeichnungspflicht in Berlin sowie die geplante Einführung in anderen Bundesländern:

Auch in der Bundesrepublik setzt sich die Kennzeichnungspflicht immer mehr durch. In Berlin sieht ein CDU-Innensenator Henkel nicht das geringste Problem, die unter Rot-Rot eingeführte individuelle Kennzeichnung fortzuführen. Von Problemen oder Beschwerden ist nichts zu hören. Nordrhein-Westfalen, Baden-Württemberg und Rheinland-Pfalz werden bei der Einführung der Kennzeichnung in Kürze folgen. (B. Peters [Grüne], Landtag Schleswig-Holstein 2012, S. 350)

Innenminister Breitner (SPD) begründete den Richtungswechsel seiner Partei in derselben Debatte ebenfalls mit der problemlosen Einführung in Berlin und Brandenburg (Landtag Schleswig-Holstein 2012, S. 358). In den Landtagsdebatten zum Thema finden sich zwar auch vereinzelte Verweise von Mitgliedern der Regierungsfraktionen auf die Vorfälle von Polizeigewalt in Berlin und in Stuttgart (Landtag Schleswig-Holstein 2012, S. 348-349). Die positiven Erfahrungen aus anderen Bundesländern mit der Kennzeichnungspflicht spielen bei der Rechtfertigung der Reform allerdings eine deutlich prominentere Rolle.

Auf Schleswig-Holstein, wo die Kennzeichnungspflicht im Dezember 2012 per Erlass angeordnet wurde, folgte knapp ein Jahr später Rheinland-Pfalz. Nach den Landtagswahlen im März 2011 musste sich die SPD, die zuvor allein regiert hatte, die Regierungsmacht mit den Grünen teilen. Wie in Schleswig-Holstein befand sich damit ein Agenda-Setter in der Regierung - und wie im hohen Norden gelang es den Grünen die Einführung der Kennzeichnungspflicht im Koalitionsvertrag festzuschreiben (SPD und Grüne 2011, S. 81). Auch in Rheinland-Pfalz verteidigte die SPD ihre Unterstützung des Vorhabens gegen Angriffe aus der CDU mit den positiven Erfahrungen in Berlin: „Fragen Sie mal bei den Kolleginnen und Kollegen dort nach, welche Probleme sie haben. - Keine“ (C. Pörksen [SPD], Landtag Rheinland-Pfalz 2013, S. 3580). Schließlich zeigte sich in diesem Fall auch der letztlich beschränkte Einfluss der Polizeigewerkschaften auf die Entscheidung. Aufgrund ihres Widerstandes kam es im Juli 2013 zur Anrufung der regierungsinternen Einigungsstelle. Zwar votierte dieses Gremium im Sinne der Polizeigewerkschaften gegen eine Kennzeichnungspflicht (Rhein-Zeitung 2013), SPD-Innenminister Lewentz setzte sich jedoch über das rechtlich nicht bindende Votum hinweg und erließ im November 2013 eine Dienstverordnung, die die individuelle Kennzeichnung verpflichtend machte (Regler 2016, S. 52). Der Fall Rheinland-Pfalz bestätigt damit auf exemplarische Weise, dass Polizeigewerkschaften die Einführung der Kennzeichnungspflicht nicht stoppen können, wenn sie keine Unterstützung von einer der Regierungsparteien erhalten.

Zusammengenommen zeigen die Fälle Schleswig-Holstein und Rheinland-Pfalz, dass nach erstmaliger Einführung der Kennzeichnungspflicht - und dem damit verbundenen Diffusionsdruck - nicht zwingend weitere Focusing Events vorliegen mussten, damit es zur Durchsetzung der Kennzeichnung in anderen Bundesländern kam. Vielmehr erleichterte der neue Status quo den Befürwortern die Durchsetzung, wenn sie in der Regierung nicht auf Gegner der Kennzeichnungspflicht trafen. 


\subsection{Hessen: Ungünstige Machtkonstellation, günstige Rahmenbedingungen}

Im Vergleich zu den bisher behandelten Fällen ist der hessische Fall insbesondere deswegen von besonderem Interesse, weil dies der einzige der erklärten Fälle ist, in dem neben einem Agenda-Setter (Grüne) auch ein Gegner der Kennzeichnungspflicht (CDU) in der Regierung vertreten war (P2). Eine genauere Betrachtung dieses Falles kann daher dabei helfen zu klären, wie der Widerstand der parteilichen Gegner der Kennzeichnungspflicht unter günstigen Rahmenbedingungen überwunden werden kann.

Wie in Berlin bildete auch in Hessen ein Focusing Event den Ausgangspunkt der politischen Debatte. In diesem Fall handelte es sich um die ,Blockupy'-Proteste gegen den Neubau der EZB in Frankfurt im Juni 2013, in deren Rahmen es zu einem massiven Polizeieinsatz inklusive der Einkesselung von knapp 1000 Demonstranten kam (FR 2013). Die Vorwürfe ungeahndeter Polizeigewalt hatten Anträge der Linken und der Grünen zur Einführung der Kennzeichnungspflicht zur Folge (Landtag Hessen 2013a, 2013b). In der parlamentarischen Debatte verwiesen Vertreter beider Parteien neben der Polizeigewalt bei den ,Blockupy'-Protesten auch auf die erfolgreiche Einführung der Kennzeichnungspflicht in Berlin, wo sich keine der zuvor von den Kennzeichnungspflicht-Gegnern vorgebrachten Befürchtungen bewahrheitet hätten. CDU-Innenminister Rhein wendete sich jedoch ebenso gegen diesen Vorstoß der beiden Oppositionsparteien wie der kleine Koalitionspartner von der FDP (Landtag Hessen 2013c, S. 10251-10258).

Noch im Schatten dieser Diskussion kam es im Herbst 2013 zum Regierungswechsel in Hessen, wobei die Grünen die FDP als Koalitionspartner der CDU ersetzten. In dieser neuen Konstellation rückte die CDU in den Koalitionsverhandlungen von ihrer ablehnenden Haltung ab und erkläre sich zur Einführung der individuellen Kennzeichnung bereit, wobei die Grünen im Gegenzug auf eine gesetzliche Regelung verzichteten (CDU und Grüne 2013, S. 38). Angesichts der weiterhin innerhalb der CDU vorherrschenden Ablehnung in dieser Frage ist es trotzdem erklärungsbedürftig, dass es im Dezember 2014 tatsächlich zur Einführung der Kennzeichnungspflicht kam (Innenministerium Hessen 2014). Regler (2015) weist in seiner ausführlichen Analyse des hessischen Falles auf zwei Ereignisse hin, die dazu beitrugen, verbleibende Widerstände aus Reihen der CDU zu beseitigen. Zum einen urteilte das Frankfurter Verwaltungsgericht Anfang Dezember 2014, dass es bei den ,Blockupy '-Protesten zu einem rechtswidrigen Einsatz von Pfefferspray seitens der Polizei gekommen sei. Das Gericht hob außerdem hervor, dass eine Identifizierung der Verantwortlichen aufgrund der fehlenden individuellen Kennzeichnungspflicht nicht möglich war - ein Problem, das auch Innenminister Beuth (CDU) anerkannte (FR 2014). Zum anderen warf mit der Neueröffnung der EZB im März 2015 ein polizeiliches Großereignis seine Schatten voraus. Nach der Eskalation bei den vorangegangenen Protesten stellte die Einführung der Kennzeichnungspflicht in diesem Kontext eine vertrauensbildende Maßnahme dar:

Die Kennzeichnungspflicht für die Polizeibeamtinnen und Polizeibeamten ist bei dem, was uns in absehbarer Zeit an besonderen Demonstrationslagen, insbesondere in Frankfurt um die Einweihung der EZB, bevorstehen wird, eine 
echte Chance, zu gewinnen, und zwar völlig egal, wie ich das persönlich sehe. Es ist offensichtlich so, dass es einen großen Teil von Menschen gibt, die zumindest als eine Erwartung gegenüber der Polizei formulieren, dass sie deutlich macht, wer es ist - ob das mit einer Nummer oder mit einer Kennzeichnung oder wie auch immer geschieht. [...] Das ist auch der Grund, warum wir den Einstieg jetzt wählen wollen und müssen. Denn wenn es sinnvoll ist, dann ist es das jetzt im Moment. (Innenminister P. Beuth [CDU], Landtag Hessen 2014, S. 1180-1181)

Nach den ,Blockupy“-Protesten im Juni 2013 existierte mit der bevorstehenden Neueröffnung der EZB im Frühjahr 2015 also eine Art zweites Focusing Event, das dafür sorgte, dass die Kennzeichnungspflicht auf der politischen Agenda blieb. In Verbindung mit dem spürbaren Diffusionsdruck herrschten damit günstige Rahmenbedingungen, die es den Grünen als Agenda-Setter letzten Endes ermöglichten, die Widerstände des Blockierers CDU innerhalb der Landesregierung zu überwinden und die Einführung der Kennzeichnungspflicht durchzusetzen.

\subsection{Mecklenburg-Vorpommern: Kennzeichnungspflicht ohne Agenda-Setter?}

Abschließend erfolgt eine genauere Betrachtung des abweichenden Falles Mecklenburg-Vorpommern, weil es dort Ende des Jahres 2017 unter einer Koalition aus SPD und CDU, also ohne Beteiligung eines unserer Agenda-Setter, zur Einführung der Kennzeichnungspflicht kam. Dieses Ergebnis ist umso erstaunlicher, wenn berücksichtigt wird, dass SPD und CDU seit 2006 zusammen die Landesregierung bildeten und dass sich ursprünglich neben der CDU, die über den gesamten Zeitraum mit Lorenz Caffier den Innenminister stellte, auch die Sozialdemokraten gegen die Kennzeichnungspflicht aussprachen. Vor diesem Hintergrund stellt sich die Frage, wie es in Mecklenburg-Vorpommern zur Einführung der Kennzeichnungspflicht kommen konnte und welche Rolle die SPD in diesem Prozess spielte.

Bei der Untersuchung der Position der mecklenburgischen SPD zum Thema Kennzeichnungspflicht lohnt es sich zu analysieren, warum sich die Sozialdemokraten zunächst gegen die Einführung einer individuellen Kennzeichnungspflicht in Mecklenburg-Vorpommern aussprachen:

In geschlossenen Einsätzen, die oft länderübergreifend stattfinden, muss es eine bundeseinheitliche Regelung geben. [...] Ein Sonderweg Mecklenburg-Vorpommerns kann dazu führen, dass wir von Polizisten anderer Länder in Großlagen nicht mehr unterstützt werden. (SPD Mecklenburg-Vorpommern 2010)

Die SPD lehnte eine Form der individuellen Kennzeichnung für Polizisten also nicht grundsätzlich ab, sondern verwies auf Probleme die daraus entstehen könnten, wenn Mecklenburg-Vorpommern in dieser Frage eine Vorreiterrolle einnehmen würde. Mit der Einführung der Kennzeichnungspflicht in anderen, auch benachbarten Bundesländern ist ein Wandel bei den Sozialdemokraten festzustellen. Im Oktober 2014 wurde ein Antrag der Grünen auf Prüfung der Einführung der Kennzeichnungspflicht zwar mit den Stimmen der SPD abgelehnt. Allerdings stimmte die SPD in erster Linie aus Rücksicht auf den Koalitionspartner CDU gegen den Antrag der 
Grünen, der lediglich von den Linken unterstützt wurde (Landtag Mecklenburg-Vorpommern 2014, S. 30-49). In der Parlamentsdebatte machte die SPD die Aufgabe ihrer ablehnenden Haltung aber ebenso klar wie in einer späteren Pressemitteilung:

In mehreren Bundesländern besteht eine Kennzeichnungspflicht. Auch das CDU-geführte Hessen wird in Kürze eine solche Regelung einführen. In Brandenburg war die CDU vor einigen Jahren sogar Initiator eines Gesetzesentwurfs zur Kennzeichnungspflicht von Polizeibeamtinnen und Polizeibeamten. Schon deshalb kann eine Kennzeichnungspflicht nicht von vornherein als ,Teufelszeug" abgetan werden. Die SPD-Fraktion wird bei unserem Koalitionspartner dafür werben, sich einer Debatte zu öffnen. Aus unserer Sicht gilt es, die Pround-Kontra-Argumente sorgfältig abzuwägen und die Erfahrungen anderer Bundesländer gründlich auszuwerten. (SPD Mecklenburg-Vorpommern 2014)

Die offenere Haltung zur Kennzeichnungspflicht wird also mit ihrer zwischenzeitlichen Einführung in anderen Bundesländern begründet. Das im Vergleich zu 2010 deutlich veränderte Argumentationsmuster der SPD stellt damit ein klares Indiz für das Auftreten von Policy-Diffusion dar.

In die Koalitionsverhandlungen mit einer bei den Landtagswahlen 2016 geschwächten CDU ging die SPD schließlich als Befürworter einer Kennzeichnungspflicht - letztlich mit Erfolg. Die Einführung der Kennzeichnungspflicht wurde gegen alle Bedenken seitens der CDU im neuen Koalitionsvertrag verankert (SPD und CDU 2016, S. 65) und im Dezember 2017 per Dienstvorschrift eingeführt (Innenministerium Mecklenburg-Vorpommern 2017). Der abweichende Fall MecklenburgVorpommerns lässt sich also damit erklären, dass die SPD nach einem durch PolicyDiffusion begünstigten inhaltlichen Positionswechsel anders als in anderen Bundesländern selbst als Agenda-Setter auftrat, der sich letztlich gegen den ebenfalls an der Regierung beteiligten Blockierer CDU durchsetzte.

Die Untersuchung des abweichenden Falles Mecklenburg-Vorpommern deutet darauf hin, dass der Positionierung der SPD eine größere Bedeutung zukommt, als im Rahmen der QCA angenommen - eine Einschätzung, die durch einen abschließenden kursorischen Blick in andere Bundesländer bestätigt wird. So war es sowohl in Baden-Württemberg (2011-16) als auch in Niedersachsen (2013-17) - dem abweichenden Fall innerhalb des dritten Lösungsterms - die SPD, die trotz gegenteiliger Vereinbarungen im Koalitionsvertrag mit dem grünen Regierungspartner (Grüne und SPD 2011, S. 66; SPD und Grüne 2013, S. 17), die Einführung der Kennzeichnungspflicht verzögerte und letztlich verhinderte. In Baden-Württemberg war dies sogar trotz des Focusing Events ,Stuttgart 21 ' der Fall (Regler 2015, S. 24; Staff und Wenzelburger 2017, S. 114-116). Auch in Hamburg waren es die Sozialdemokraten, die nach 2015 gegen den Willen des grünen Koalitionspartners die Kennzeichnungspflicht blockierten. Erst ein Focusing Event in Form der Polizeigewalt beim G20Gipfel bereitete dieser Blockadehaltung ein Ende (Taz 2019). In Sachsen-Anhalt schließlich wandelte sich die SPD ähnlich wie in Mecklenburg-Vorpommern sogar zum Befürworter und sorgte 2017 im Verbund mit dem grünen Koalitionspartner (und gegen den ursprünglichen Widerstand der ebenfalls an der Regierung beteiligten CDU) für die Einführung der Kennzeichnungspflicht (Landtag Sachsen-Anhalt 2017, S. 57-65). Der vorangehende Gesinnungswandel innerhalb der SPD resultier- 
te aus einem Mitgliederentscheid, der wiederum durch ein lokales, in das Jahr 2012 zurückreichendes Focusing Event ausgelöst wurde. ${ }^{18}$

In der Gesamtbetrachtung dieser Fälle zeigt sich somit, dass die SPD nicht zwangsläufig dem Koalitionspartner folgt, sondern in einigen Bundesländern einen aktiven Einfluss auf das Outcome nimmt. Die Haltung der SPD ist dabei weder über Ländergrenzen hinweg noch über die Zeit stabil. Angesichts eines steigenden Diffusionsdrucks und unter dem Eindruck von Focusing Events ist aber eine Tendenz zur Befürwortung der Kennzeichnungspflicht feststellbar, sodass sich die SPD in einigen Fällen zum Agenda-Setter wandelte.

\section{Fazit und Ausblick}

Das Ziel dieses Artikels war es zu klären, warum einige Bundesländer eine verpflichtende Kennzeichnung für Polizisten in geschlossenen Einheiten eingeführt haben, während dies in anderen Bundesländern bisher nicht geschehen ist. Den Startpunkt unserer Untersuchung bildeten dabei zwei Grundannahmen, nämlich dass Parteien einen wichtigen Erklärungsfaktor darstellen und dass es das Zusammenspiel von Parteifärbung der Regierung und günstigen Rahmenbedingungen ist, die zur Einführung der Kennzeichnungspflicht führen. Die Ergebnisse der QCA und der Fallstudien bestätigen diese Annahmen und erlauben uns zum Abschluss eine detaillierte Beantwortung der Forschungsfrage.

Die QCA hat demonstriert, dass das Vorhandensein eines Agenda-Setters eine notwendige Bedingung darstellt und damit von zentraler Bedeutung für die Einführung der Kennzeichnungspflicht ist. So waren mit einer Ausnahme in allen Ländern, in denen die Kennzeichnung eingeführt wurde, zu diesem Zeitpunkt entweder die Grünen und/oder die Linken an der Regierung beteiligt. Die Ausnahme bildet MecklenburgVorpommern. Für diesen abweichenden Fall konnte in einer zusätzlichen Fallstudie gezeigt werden, dass dort die SPD als Agenda-Setter auftrat. Darüber hinaus lässt sich auf Basis der QCA festhalten, dass das Auftreten eines Agenda-Setters bei gleichzeitigem Fehlen eines Blockierers eine günstige Regierungskonstellation für die Einführung der Kennzeichnungspflicht darstellt. Die QCA und die angeschlossenen Fallstudien zeigen aber auch, dass die parteipolitische Zusammensetzung der Regierung als Erklärungsfaktor alleine nicht ausreicht, sondern dass zusätzlich die Rahmenbedingungen, unter denen Regierungsparteien operieren, berücksichtigt werden müssen. Dabei hat sich gezeigt, dass Focusing Events und Policy-Diffusion die Einführung der Kennzeichnungspflicht begünstigen. Im Falle des Vorreiters Berlin war das Auftreten eines Focusing Events von zentraler Bedeutung, weil die Befürworter der Kennzeichnungspflicht in diesem Fall nicht auf Erfahrungen aus anderen Bundesländern verweisen konnten. Die Einführung in Berlin erleichterte Befürwortern in anderen Ländern daraufhin die Durchsetzung der Kennzeichnungs-

\footnotetext{
18 Im August 2012 war ein Teilnehmer einer Demonstration in Halle von einem Polizisten schwer verletzt worden, ohne dass der Verantwortliche später identifiziert werden konnte. Daraufhin kam es auf Initiative der Jusos zu einem Mitgliederentscheid, in dem sich eine klare Mehrheit der SPD-Mitglieder für eine individuelle Kennzeichnungspflicht von Polizisten aussprach (Mitteldeutsche Zeitung 2013).
} 
pflicht, auch wenn kein unmittelbares Focusing Event vorlag. Der hessische Fall hat schließlich demonstriert, dass unter sehr günstigen Rahmenbedingungen (Focusing Event+ Diffusion) auch die Vetomacht eines an der Regierung beteiligten Gegners der Kennzeichnung, in diesem Fall der CDU, gebrochen werden kann.

Anhand unserer Befunde lässt sich abschließend eine vorsichtige Prognose für die Bundesländer abgeben, in denen es keine individuelle Kennzeichnung für Polizisten gibt. Abgesehen von Hamburg, wo die rot-grüne Regierung nach dem Focusing Event des G20-Gipfels die Kennzeichnung für 2019 ankündigte, erscheint eine Einführung in den anderen Ländern unter den aktuellen Landesregierungen unwahrscheinlich. So ist nur in Baden-Württemberg mit den Grünen ein Agenda-Setter an der Regierung beteiligt - die grün-schwarze Regierung hat die Einführung für die aktuelle Legislaturperiode allerdings ausgeschlossen. In den verbleibenden Ländern finden sich keine Agenda-Setter, dafür aber Gegner der Kennzeichnungspflicht in der Regierung: Bayern (CDU, Freie Wähler), Nordrhein-Westfalen (CDU, FDP), Niedersachsen, Saarland und Sachsen (jeweils CDU und SPD). ${ }^{19}$ Die Kennzeichnungspflicht dürfte in diesen Ländern somit frühestens nach Regierungswechseln wieder auf die politische Agenda kommen, insbesondere wenn sich die Befürworter bei ihren Vorstößen auf neue Focusing Events stützen können. Schließlich ist auch eine Abschaffung der Kennzeichnungspflicht unter umgekehrten Vorzeichen nicht gänzlich ausgeschlossen, wie der nordrhein-westfälische Fall verdeutlicht. Dort machte die aktuelle Koalition aus CDU und FDP - in Nordrhein-Westfalen beides erklärte Gegner der Kennzeichnung - die gesetzliche Regelung ihrer rot-grünen Vorgänger im Oktober 2017 rückgängig.

Danksagung Wir danken Thomas Rixen, Lukas Hakelberg, Leo Ahrens und Simon Linder für ihre zahlreichen Anregungen und Kommentare sowie den zwei anonymen Gutachtern für ihre konstruktive Kritik. Besonderer Dank gebührt zudem Nikolaus Jopke für seine wertvollen Ratschläge in methodischen Fragen sowie Svenja Schrader und Lea Maurer für ihre Unterstützung bei der Datenerhebung und -aufbereitung.

Funding Open Access funding provided by Projekt DEAL.

Open Access Dieser Artikel wird unter der Creative Commons Namensnennung 4.0 International Lizenz veröffentlicht, welche die Nutzung, Vervielfältigung, Bearbeitung, Verbreitung und Wiedergabe in jeglichem Medium und Format erlaubt, sofern Sie den/die ursprünglichen Autor(en) und die Quelle ordnungsgemäß nennen, einen Link zur Creative Commons Lizenz beifügen und angeben, ob Änderungen vorgenommen wurden.

Die in diesem Artikel enthaltenen Bilder und sonstiges Drittmaterial unterliegen ebenfalls der genannten Creative Commons Lizenz, sofern sich aus der Abbildungslegende nichts anderes ergibt. Sofern das betreffende Material nicht unter der genannten Creative Commons Lizenz steht und die betreffende Handlung nicht nach gesetzlichen Vorschriften erlaubt ist, ist für die oben aufgeführten Weiterverwendungen des Materials die Einwilligung des jeweiligen Rechteinhabers einzuholen.

Weitere Details zur Lizenz entnehmen Sie bitte der Lizenzinformation auf http://creativecommons.org/ licenses/by/4.0/deed.de.

${ }_{19}$ Der sich nach der Wahl in Sachsen abzeichnende Regierungswechsel zu einer Koalition aus CDU, Grünen und SPD dürfte aufgrund der Beteiligung der Grünen die Chancen der Einführung einer Kennzeichnungspflicht erhöhen. 


\section{Anhang}

Tab. 3 Analyse notwendiger Bedingungen

\begin{tabular}{llll}
\hline Konditionen & Konsistenz & Abdeckung & $\begin{array}{l}\text { RoN-Wert (Relevance of Neces- } \\
\text { sity) }\end{array}$ \\
\hline$\sim$ Blockierer+ Diffusion & 1 & 0,385 & 0,448 \\
Agenda-Setter & 0,9 & 0,529 & 0,733 \\
Focusing Event + Diffusion & 0,9 & 0,375 & 0,5 \\
$\sim$ Focusing Event + Diffusion & 0,9 & 0,25 & 0,1 \\
$\sim$ Blockierer+ $\sim$ Focusing Event & 0,9 & 0,25 & 0,1 \\
Blockierer+ $\sim$ Focusing Event & 0,9 & 0,25 & 0,1 \\
$\sim$ Focusing Event + Diffusion & 0,9 & 0,25 & 0,1 \\
$\sim$ Blockierer+ $\sim$ Agenda + Focusing & 0,9 & 0,257 & 0,133 \\
Event & & & \\
\hline$\sim \sim$ & &
\end{tabular}

, “ kennzeichnet jeweils die Negation einer Kondition

„+" steht für ein logisches ,oder“ 


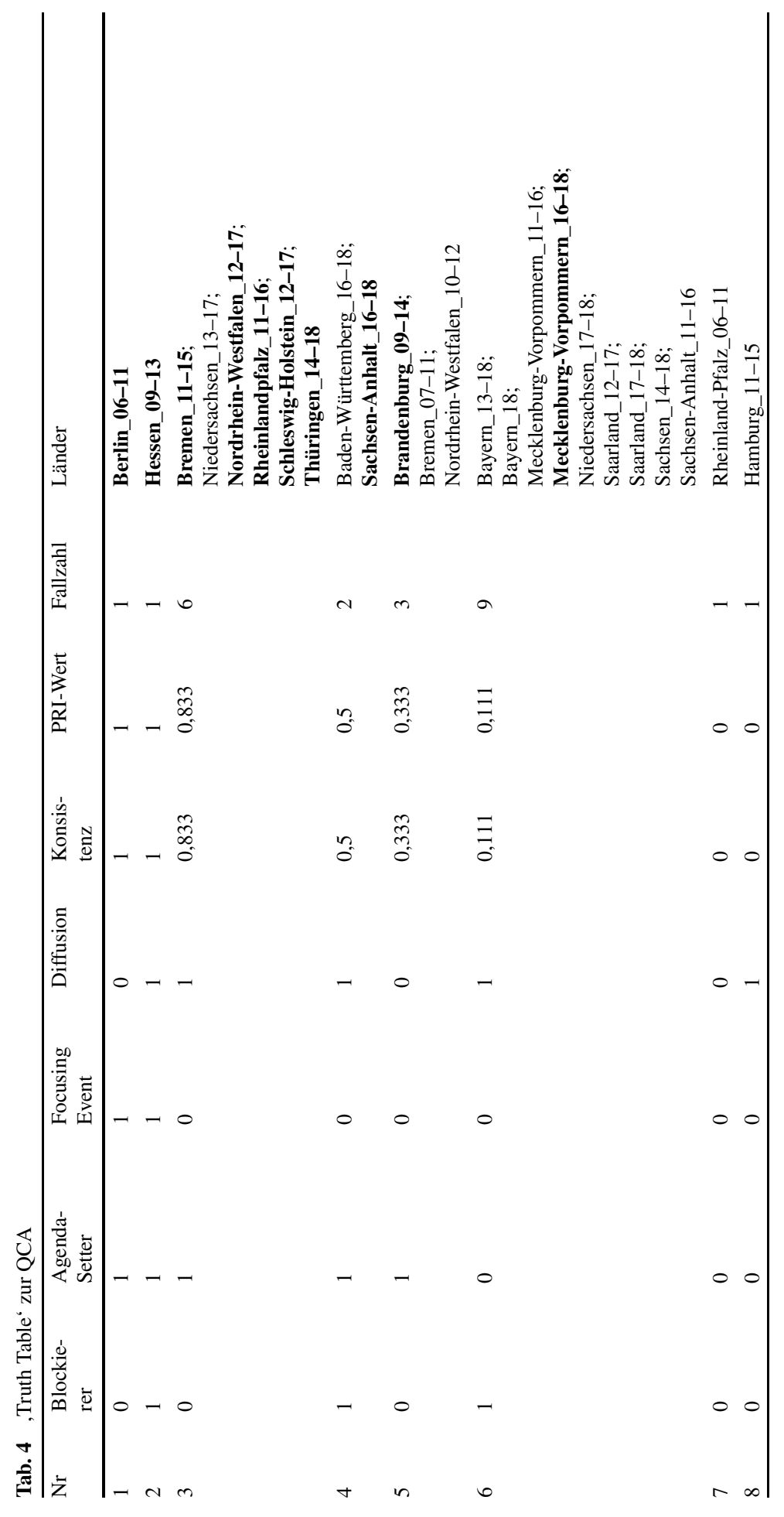




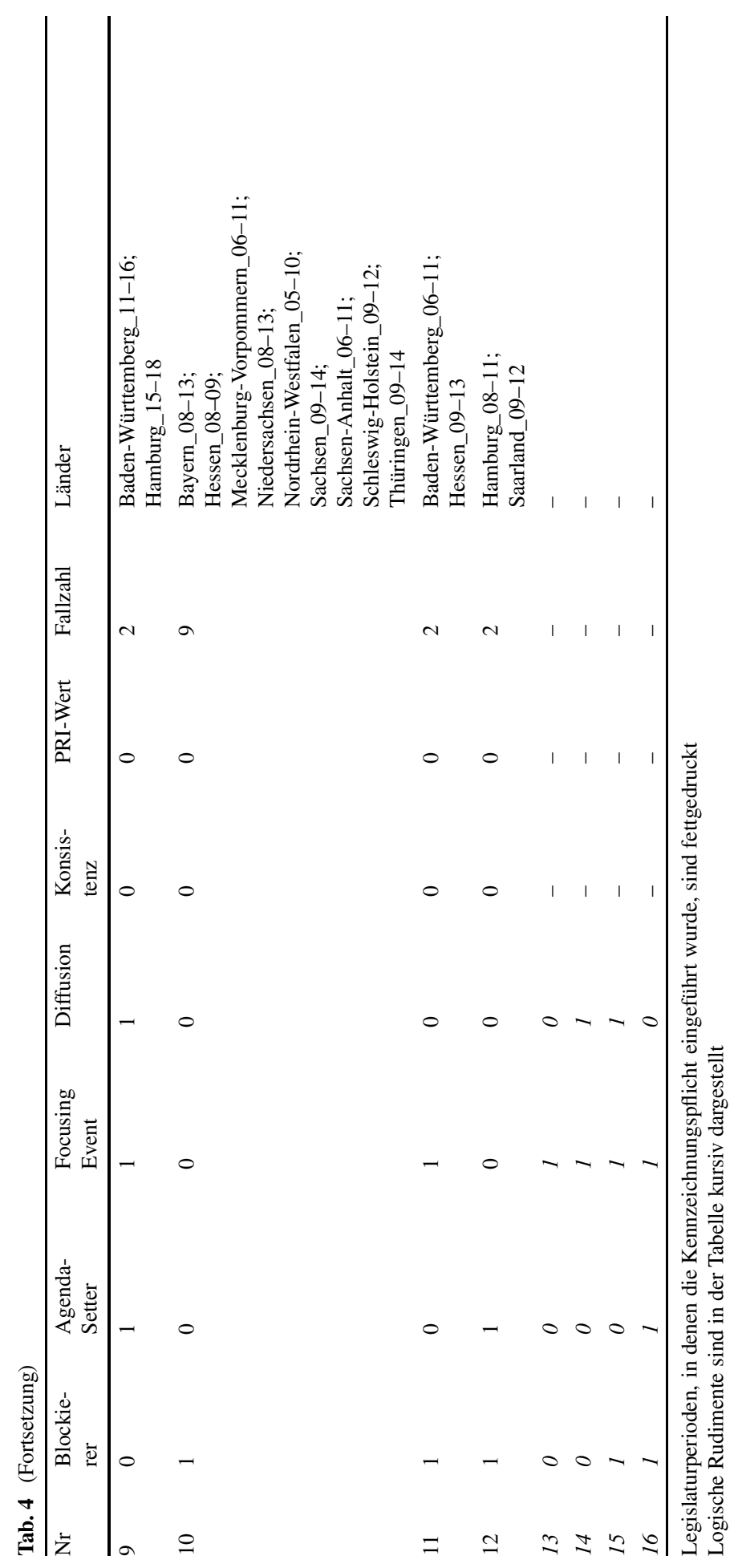




\section{Literatur}

Abgeordnetenhaus Berlin. 2007. Plenarprotokoll 16/7, 22. Februar 2007.

Aden, Hartmut. 2010. Die Kennzeichnung von Polizeibediensteten. Die Polizei 101(12):347-352.

Aden, Hartmut. 2012. Polizeikennzeichnung - Konfliktlinien und Akteurskoalitionen in einer jahrzehntelangen Debatte. In Empirische Polizeiforschung XIV: Polizei und Gewalt. Interdisziplinäre Analysen zu Gewalt gegen und durch Polizeibeamte, Hrsg. Thomas Ohlemacher, Jochen-Thomas Werner, 143-158. Frankfurt/Main: Verlag für Polizeiwissenschaft.

Amnesty International. 2010. Täter unbekannt. Mangelnde Aufklärung von mutmaßlichen Misshandlungen durch die Polizei in Deutschland. Berlin: Amnesty International.

Barczak, Tristan. 2011. Die Kennzeichnungspflicht für Polizeibeamte im Lichte des Verfasungsrechts. Neue Zeitschrift für Verfassungsrecht 30(14):852-855.

Barczak, Tristan. 2014. Die Kennzeichnungspflicht für Polizeibeamte in Berlin und Brandenburg. LKV 24(9):391-395.

Beinborn, Niclas, Stephan Grohs, Renate Reiter, und Nicolas Ullrich. 2018. Eigenständige Jugendpolitik‘. Varianz in den Ländern. Zeitschrift für Vergleichende Politikwissenschaft 12(4):743-762.

Birkel, Christoph. 2008. Gewaltkriminalität: Zur Bedeutung von Politikproduktion und parteipolitischer Färbung der Landesregierungen. In Die Politik der Bundesländer. Staatstätigkeit im Vergleich, Hrsg. Achim Hildebrandt, Frieder Wolf, 89-112. Wiesbaden: VS.

Blatter, Joachim, und Markus Haverland. 2012. Designing case studies. Explanatory approaches in small-N research. London: Palgrave Macmillan.

Braun, Dietmar, und Fabrozio Gilardi. 2006. Taking 'Galton's problem' seriously. Journal of Theoretical Politics 18(3):298-322.

CDU, und Grüne. 2013. Verlässlich gestalten - Perspektiven eröffnen. Hessen 2014 bis 2019

Der Tagesspiegel. 2007. „Wir haben wieder eine Zunahme der Rohheitsdelikte“. 15. Dezember. https:// www.tagesspiegel.de/berlin/berliner-polizeipraesident-glietsch-wir-haben-wieder-eine-zunahmeder-rohheitsdelikte/1121480.html. Zugegriffen: 6. Feb. 2020.

Diederichs, Otto. 2009. Never Ending Story. Kennzeichnung von Polizeibeamtinnen. Bürgerrechte \& Polizei/CILIP 94:58-65.

Drescher, Falko. 2014. Polizisten außer Kontrolle? Zur Diskussion über die Notwendigkeit einer Überwachung der Polizei. Hamburg: Disserta.

Dusa, Adrian. 2017. QCA with R. A comprehensive resource. New York: Springer.

FDP Hamburg. 2018. Kennzeichnungspflicht für Polizisten endlich einführen. https://www.fdp-fraktionhh.de/kennzeichnungspflicht-fuer-polizisten-endlich-einfuehren/. Zugegriffen: 6. Feb. 2020.

Fiss, Peer C. 2011. Building better causal theories. A fuzzy set approach to typologies in organization research. Academy of Management Journal 54(2):393-420.

FR (Hrsg.). 2013. Entsetzen über Polizei-Kessel, Frankfurter Rundschau, 6. Juni 2013. https://www.fr. de/frankfurt/blockupy-sti58236/entsetzen-ueber-polizei-kessel-11282282.html. Zugegriffen: 6. Feb. 2020.

FR (Hrsg.). 2014. Nummern für Polizisten, Frankfurter Rundschau, 11. Dezember 2014. https://www.fr. de/rhein-main/cdu-org26591/nummern-polizisten-11195450.html. Zugegriffen: 6. Feb. 2020.

Frevel, Bernhard, und Hermann Groß. 2008. „Polizei ist Ländersache!“ - Politik der Inneren Sicherheit. In Die Politik der Bundesländer. Staatstätigkeit im Vergleich, Hrsg. Achim Hildebrandt, Frieder Wolf, 67-88. Wiesbaden: VS.

Frevel, Bernhard, und Hermann Groß. 2016. „Polizei ist Ländersache!“ - Polizeipolitik unter den Bedingungen des deutschen Föderalismus. In Die Politik der Bundesländer. Zwischen Föderalismusreform und Schuldenbremse, Hrsg. Achim Hildebrandt, Frieder Wolf, 61-86. Wiesbaden: Springer VS.

Grüne, und SPD. 2011. Der Wechsel beginnt. Koalitionsvertrag zwischen BÜNDNIS 90/DIE GRÜNEN und der SPD Baden-Württemberg

Günther, Wolfgang, Dennis Kurrek, und Annette E. Töller. 2019. Ein starker Fall für die Parteiendifferenztheorie. Die Einführung der Gesundheitskarte für Asylsuchende in den Bundesländern. Zeitschrift für Politikwissenschaft 29(3):361-392.

Heinrich, Anja. 2011. Polizeikennzeichnung in Berlin und Brandenburg. Mitteilungen - Zeitschrift für Aufklärung und Bürgerrechte 214:10-11.

Hörisch, Felix. 2018. Asylpolitik im Bundesländervergleich. Eine fuzzy-set Qualitative Comparative Analysis. Zeitschrift für Vergleichende Politikwissenschaft 12(4):783-803.

Innenministerium Hessen. 2014. Neue Körperschutzausstattungen für hessische Polizeibeamtinnen und -beamten, Hessisches Ministerium des Inneren und für Sport, 15. Dezember 2014. https:// 
innen.hessen.de/pressearchiv/pressemitteilung/neue-koerperschutzausstattungen-fuer-hessischepolizeibeamtinnen-und-beamten. Zugegriffen: 6. Feb. 2020.

Innenministerium Mecklenburg-Vorpommern. 2017. Verwaltungsvorschrift zur Individuellen Kennzeichnung von Polizistinnen und Polizisten in geschlossenen Einheiten, Ministerium für Inneres und Europa, 19. Dezember 2017. https://www.regierung-mv.de/Landesregierung/im/Aktuell/?id=134061\& processor=processor.sa.pressemitteilung. Zugegriffen: 6. Feb. 2020.

Jahn, Detlef. 2015. Diffusion. In Handbuch Policy-Forschung, Hrsg. Georg Wenzelburger, Reimut Zohlnhöfer, 247-276. Wiesbaden: Springer VS.

Kingdon, John W. 1995. Agendas, alternatives, and public policies. New York: Longman.

Knaust, Maik. 2017. Letzte Bastionen anonymer Polizei - Bedarf es einer Reform des niedersächsischen Polizeirechts im Hinblick auf eine Kennzeichnungspflicht für Polizeibeamtinnen und Polizeibeamte? Deutsches Verwaltungsblatt 132(14):876-882.

Landtag Hessen. 2013a. Antrag der Fraktion DIE LINKE betreffend Beamtenkennzeichnung, Drs. 18/7509, 17. Juni 2013

Landtag Hessen. 2013b. Gesetzentwurf der Fraktion BÜNDNIS90/DIE GRÜNEN für ein zehntes Gesetz zur Änderung des Hessischen Gesetzes über die öffentliche Sicherheit und Ordnung (HSOG), Drs. 18/7522, 18. Juni 2013

Landtag Hessen. 2013c. Plenarprotokoll 18/143, 26. Juni 2013

Landtag Hessen. 2014. Plenarprotokoll 19/18, 16. Juli 2014

Landtag Mecklenburg-Vorpommern. 2014. Plenarprotokoll 6/78, 16. Oktober 2014

Landtag Rheinland-Pfalz. 2013. Plenarprotokoll 16/55, 19. September 2013

Landtag Sachsen-Anhalt. 2017. Stenografischer Bericht 7/28, 20. Juni 2017

Landtag Schleswig-Holstein. 2010a. Entwurf eines Gesetzes zur Änderung des Allgemeinen Verwaltungsgesetzes für das Land Schleswig-Holstein, Drs. 17/251, 10. Februar 2010

Landtag Schleswig-Holstein. 2010b. Plenarprotokoll 17/11, 24. Februar 2010

Landtag Schleswig-Holstein. 2012. Plenarprotokoll 18/6, 24. August 2012

Mitteldeutsche Zeitung. 2013. SPD will nummerierte Polizisten, Mitteldeutsche Zeitung, 6. Januar 2013. https://www.mz-web.de/mitteldeutschland/sachsen-anhalt-spd-will-nummerierte-polizisten6952858. Zugegriffen: 6. Feb. 2020.

Peter, Tobias. 2011. Namens- oder Nummernschilder. Erneute Debatte, alte Argumente. Bürgerrechte \& Polizei/CILIP 99:15-22.

Polizeipräsident Berlin. 2010. Geschäftsanweisung ZSE Nr. 2/2009 über das Tragen von Namensschildern, 26. November 2010

Ragin, Charles C. 2000. Fuzzy-set social science. Chicago: University of Chicago Press.

Ragin, Charles C. 2008. Redesigning social inquiry: fuzzy sets and beyond. Chicago: University of Chicago Press.

Regler, Andreas. 2015. Die Einführung der Kennzeichnungspflicht in Hessen. Polizei \& Wissenschaft 4:13-26.

Regler, Andreas. 2016. Die Kennzeichnungspflicht geschlossener Polizeieinheiten. Eine Analyse der Situation in Deutschland unter besonderer Berücksichtigung der parteipolitischen Standpunkte. Zeitschrift für Polizeiwissenschaft und polizeiliche Praxis 4:49-61.

Rhein-Zeitung (Hrsg.). 2013. Polizei: Verwaltungsrichter hält Kennzeichnung für überflüssig, RheinZeitung, 8. August 2013. https://www.rhein-zeitung.de/region_artikel,-polizei-verwaltungsrichterhaelt-kennzeichnung-fuer-ueberfluessig-_arid,1020765.html. Zugegriffen: 6. Feb. 2020.

Rowe, Carolyn, und Ed Turner. 2016. Justice and prisons policy. In Die Politik der Bundesländer. Zwischen Föderalismusreform und Schuldenbremse, Hrsg. Achim Hildebrandt, Frieder Wolf, 87-113. Wiesbaden: Springer VS.

Sack, Detlef, und Katharina Sarter. 2018. Policy-Varianz durch Europäisierung? Zur Wettbewerbs- und Vergabepolitik der deutschen Länder. Zeitschrift für Vergleichende Politikwissenschaft 12(4):725-742.

Sack, Detlef, und Annette E. Töller. 2018. Einleitung: Policies in den deutschen Ländern. Zeitschrift für Vergleichende Politikwissenschaft 12(4):603-619.

Schneider, Carsten Q. 2018. Realists and idealists in QCA. Political Analysis 26(2):246-254.

Schneider, Carsten Q., und Ingo Rohlfing. 2013. Combining QCA and process tracing in set-theoretic multi-method research. Sociological Methods \& Research 42(4):559-597.

Schneider, Carsten Q., und Claudius Wagemann. 2012. Set-theoretic methods for the social sciences. A guide to qualitative comparative analysis. Cambridge: Cambridge University Press.

Skaaning, Svend-Erik. 2011. Assessing the robustness of crisp-set and fuzzy-set QCA results. Sociological Methods \& Research 40(2):391-408. 
SPD Mecklenburg-Vorpommern. 2010. Keine Kennzeichnung von Polizisten in geschlossenen Einsätzen, 30. November 2010

SPD Mecklenburg-Vorpommern. 2014. Debatte über Einführung einer Kennzeichnungspflicht sinnvoll, 11. September 2014

SPD, und CDU. 2011. Koalitionsvereinbarung zwischen der Sozialdemokratischen Partei Deutschlands, Landesverband Berlin, und der Christlich Demokratischen Union, Landesverband Berlin

SPD, und CDU. 2016. Koalitionsvereinbarung zwischen SPD und CDU Mecklenburg-Vorpommern für die 7. Wahlperiode 2016-2021

SPD, und Grüne. 2011. Den sozial-ökologischen Wandel gestalten. Koalitionsvertrag Rheinland-Pfalz, 2011-2016

SPD, und Grüne. 2013. Erneuerung und Zusammenhalt. Nachhaltige Politik für Niedersachsen

SPD, und PDS. 2002. Koalitionsvereinbarung zwischen der Sozialdemokratischen Partei Deutschlands (SPD) und der Partei des Demokratischen Sozialismus (PDS) für die Legislaturperiode 2001-2006

SPD, Grüne, und SSW. 2012. Bündnis für den Norden. Neue Horizonte für Schleswig-Holstein. Koalitionsvertrag 2012 bis 2017 zwischen SPD, Bündnis 90/Die Grünen und dem Südschleswigschen Wählerverband

Spier, Tim. 2011. Welche politischen Einstellungen haben die Mitglieder von Parteien? In Parteimitglieder in Deutschland, Hrsg. Tim Spier, Markus Klein, Ulrich von Alemann, Hanna Hoffmann, Annika Laux, Alexandra Nonnenmacher, und Katharina Rohrbach, 121-138. Wiesbaden: VS.

Spitzer, Cordula. 2016. Persönlichkeitsschutz von Amtsträgern? Zur Kennzeichnungspflicht von Polizeibeamten. Stuttgart: Boorberg.

Staff, Helge, und Georg Wenzelburger. 2017. Innere Sicherheit und Justiz. Zwischen Großreform und Kontinuität. In Das grün-rote Experiment in Baden-Württemberg. Eine Bilanz der Landesregierung Kretschmann 2011-2016, Hrsg. Felix Hörisch, Stefan Wurster, 97-123. Wiesbaden: Springer VS.

Stoiber, Michael, und Annette E. Töller. 2016. Ursachen der Privatisierung des Maßregelvollzugs in Deutschland. Eine QCA im Bundesländervergleich. Zeitschrift für Vergleichende Politikwissenschaft 10(1):9-36.

Taz (Hrsg.). 2019. Kennzeichnungspflicht für Polizisten. Hamburg zieht Lehre aus G20, tageszeitung, 23. April 2019. https://taz.de/Kennzeichnungspflicht-fuer-Polizisten/!5589225/. Zugegriffen: 6. Feb. 2020.

Tepe, Markus, und Pieter Vanhuysse. 2013. Cops for hire? The political economy of police employment in the German states. Journal of Public Policy 33(2):165-199.

Thiem, Alrik. 2019. Beyond the facts: limited empirical diversity and causal inference in qualitative comparative analysis. Sociological Methods \& Research https://doi.org/10.1177/0049124119882463.

Thinnes, Birgit. 2014. Wege aus der Anonymität des Staates. Ein kriminologisch-empirischer Beitrag zur Kennzeichnungspflicht der Polizei. Frankfurt/Main: Verlag für Polizeiwissenschaft.

Wagemann, Claudius, und Schneider Q. Carsten. 2015. Transparency standards in qualitative comparative analysis. Qualitative \& Multi-Method Research 13:38-42.

Wenzelburger, Georg. 2015. Die Politik der Inneren Sicherheit. In Handbuch Policy-Forschung, Hrsg. Georg Wenzelburger, Reimut Zohlnhöfer, 663-698. Wiesbaden: Springer VS. 